\title{
EL PAPEL DE LA CONSTANCIA EN EL APRENDIZAJE DEL PASADO. EL RASTRO PERDIDO*
}

Amos Rapoport ***

Universidad de Wisconsin-Milwaukee

Esquema gráfico de la relación vecindario-vía arteria encontrada en India, China, Thailandia e Indonesia. Fuente: Imagen suministrada por el autor.

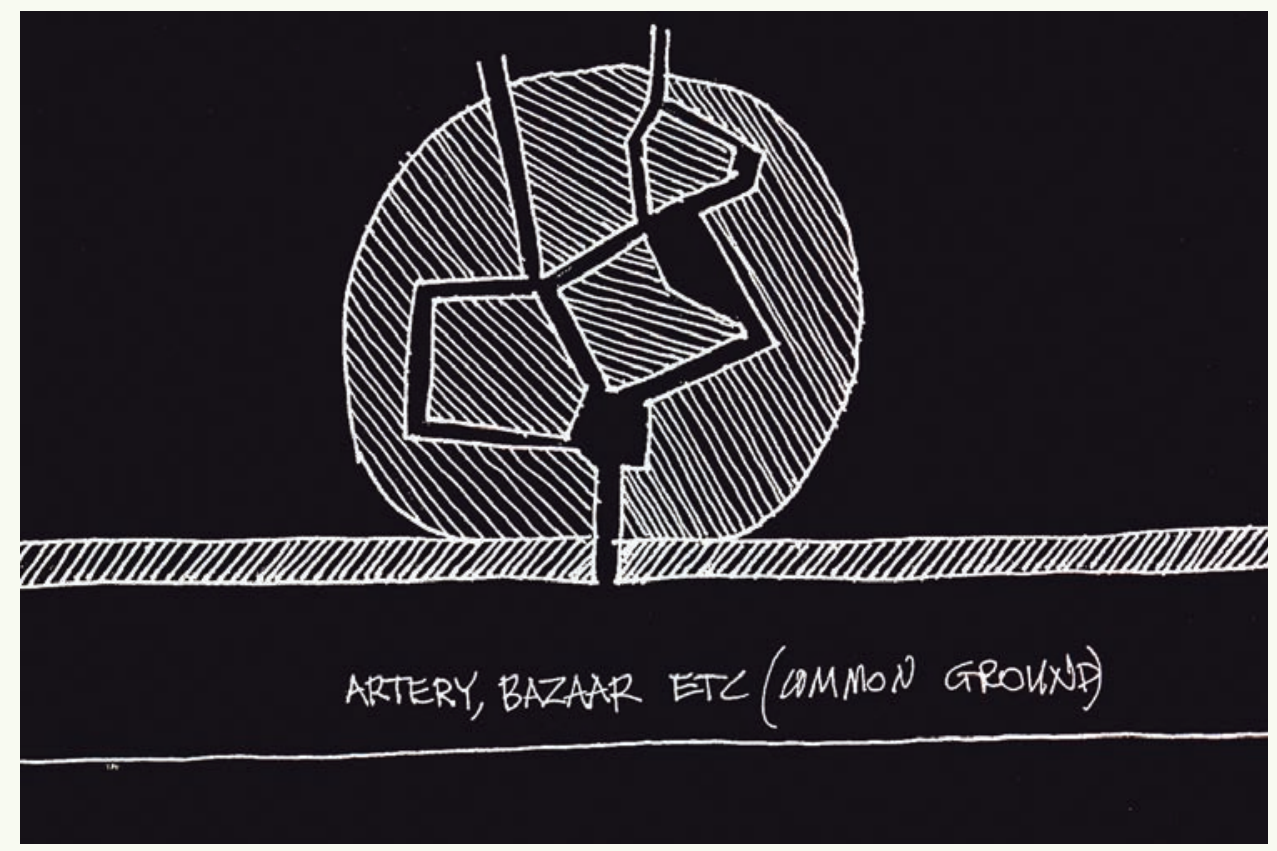

\section{RESUMEN}

En este artículo vuelvo a visitar algunos de los temas que antes he abordado, hace mucho tiempo. Necesitan reiteración porque han sido re-examinados. El tema trata esencialmente sobre aprender del pasado y de la amplia gama de entornos que conocemos. Este es un recurso increíble, un registro de cómo el ser humano ha creado ambientes, patrones y sus éxitos y fracasos, entre otros. La suposición que uno puede aprender del pasado, que hay valor en la tradición, presupone alguna constancia de las personas y el entorno. Esto se discute brevemente y el punto alcanzado es que tal aprendizaje debe estar a cierto nivel de abstracción. El trabajo a ese nivel también abre muchos campos nuevos e inesperados (y "poco probables"), se torna importante involucrar nuevos campos y sus evoluciones en la investigación EBS (Environment-Behavior Studies). Un ejemplo reciente de este tipo de campos "poco probables" se discute brevemente.

\section{PALABRAS CLAVE}

Constancia, aprendizaje, abstracción, comportamiento, ambiente 


\section{THE ROLE OF CONSTANCY IN LEARNING FROM THE PAST. A LOST TRACK}

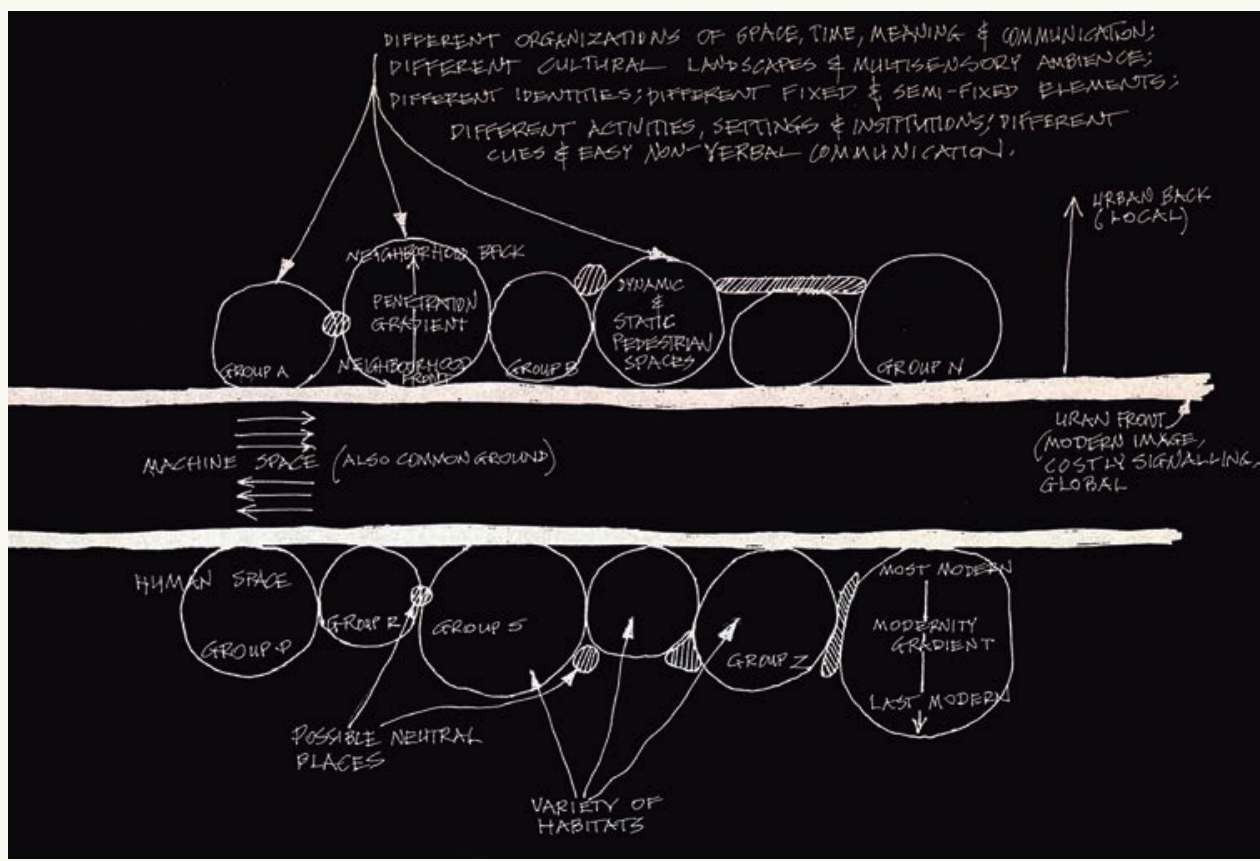

\section{ABSTRACT}

In this paper I revisit some themes I have addressed before, some a very long time ago. They need reiterating because they are being re-examined. The topic is essentially about learning from the past and from the full range of environments about which we know. These are an incredible resource, a record of how human have created environments, their successes and failure, patterns, etc. The assumption that one can learn from the past, that there is value in tradition, presupposes some constancy regarding both people and environments. This is discussed briefly and the point made that such learning need to be at some level of abstraction. Working at that level also makes relevant many new and unexpected (and "unlikely") fields, it then becomes important to involve new fields, and developments in them in EBS (Environment-Behavior Studies) research, one recent example of such on "unlikely" field is discussed briefly.

\section{KEYWORDS}

Constancy, learning, abstraction, environment, behavior
Interpretación gráfica del modelo que permite gran variedad de hábitats, con ambientes diferentes, diferentes organizaciones del espacio, tiempo. el significado y la comunicación, los colores, los sonidos, los olores, los elementos semi-fijos, y demás. Fuente: Imagen suministrada por el autor. 


\section{INTRODUCCIÓN}

Hay varias razones para volver a examinar estos temas

a. Los temas abordados se hacen más fuertes cada vez que los reviso y reciben apoyo de los resultados de nuevas investigaciones, a menudo, de nuevas disciplinas I.

b. Cada vez se unen y se refuerzan más entre sí y establecen nuevos vínculos que conducen a la generalización.

c. Esto, a su vez, conduce a muy fuertes marcos conceptuales que juegan un papel importante en el desarrollo de la (muy necesaria) teoría.

d. Los resultados de nuevas investigaciones no sólo refuerzan el argumento en que se hizo, sino que sugieren cómo el aprendizaje puede ocurrir, y cómo uno puede beneficiarse de los campos, cada vez más nuevos, usando conceptos y modelos de ellos y de la investigación en curso. Esto sólo puede ocurrir en un cierto nivel de abstracción - las lecciones son indirectas.

El supuesto que uno puede aprender de los últimos presupone cierta constancia, tanto de las personas y el entorno con respecto a (asentamientos, viviendas, espacios u otros), así como las interacciones entre ellos².

Esta constancia permite aprovechar la amplia gama de entornos construidos que conocemos (y sobre los cuales se descubre más y más) y, como han argumentado antes, esto implica una importante expansión del dominio: pues incluye la totalidad de entornos de todas las culturas, la duración completa de la historia (posiblemente incluye la arquitectura de los animales) y toda la gama de ambientes incluido asentamientos espontáneos los cuales son inadecuadamente descritos como "tugurios", los cuales, si se aborda adecuadamente, tienen mucho que enseñarnos (como lo hace lo vernáculo, lo tribal, lo popular y otros entornos) ${ }^{3}$.

Esta expansión del dominio es un recurso increíble y aún abandonado, un registro de las interacciones humanas con los entornos, sus intenciones, objetivos y metas en la creación de ambientes, éxitos y fracasos (y las razones de éstos), los patrones y procesos de regularidades, entre otros. El pasado representa un laboratorio sin precedentes si se aproxima de manera adecuada tiene mucho que enseñarnos.

Muchos de los temas discutidos se desarrollan con más detalle en un artículo reciente: "Designing for people: some implications" En: TRIALOG, No. 106. 3, 2010. pp. 4-45.

2 Téngase en cuenta, como se verá más adelante, que esta constancia, especialmente de los ambientes es, en parte, una cuestión de escala - que se aplica mayormente a pequeña escala-.

3 También puede resultar útil acercarse a ellos en términos de la idea de Patrick Gedde de "cirugía conservadora" que, por desgracia, ha sido olvidada e ignorada. Ver además: RAPOPORT, A. Human aspects of urban form, Oxford, Pergamon Press. 1977 + RAPOPORT, A. "Spontaneous settlements as vernacular design" in C.V. Patton (ed.) Spontaneus Shelter. Philadelphia, Temple University Press. 1988. pp. 5I-77 + RAPOPORT, A. The meaning of the built environment. Tucson. University of Arizona Press. 1990 + RAPOPORT, A. History and precedent in environmental design. New York. Plenum. 1990 
La lección puede ser acerca de los procesos y las características del producto ${ }^{4}$. Aquí haré hincapié en las características del producto, apoyo, lo que yo llamo equilibrio estable ${ }^{5}$, movimiento de los peatones, ambiente, complejidad, la capacidad de respuesta cultural y social $^{6}$. También hay lecciones sobre los sistemas de reglas, que pueden ser implementados mediante materiales modernos, preservado el núcleo de la cultura y proporcionada la imagen moderna apropiada (discutido más adelante) ${ }^{7}$. También se pueden identificar aspectos positivos y negativos en las calidades de los perfiles ambientales de diferentes grupos, de los diferentes actores y entender ( $y$ posiblemente resolver) los conflictos ${ }^{8}$. Es necesario reiterar que este tipo de aprendizaje, y el uso de la construcción de entornos con sistemas modelo ${ }^{9}$ sólo puede ocurrir a cierto nivel de abstracción y nunca es directo ${ }^{10}$. (Ver figura I).

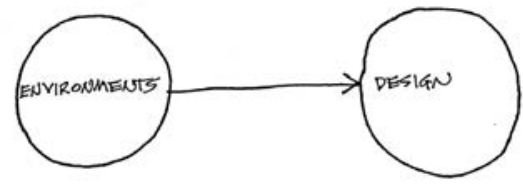

THIS IS NOT LEXRNING BUT COPMNE

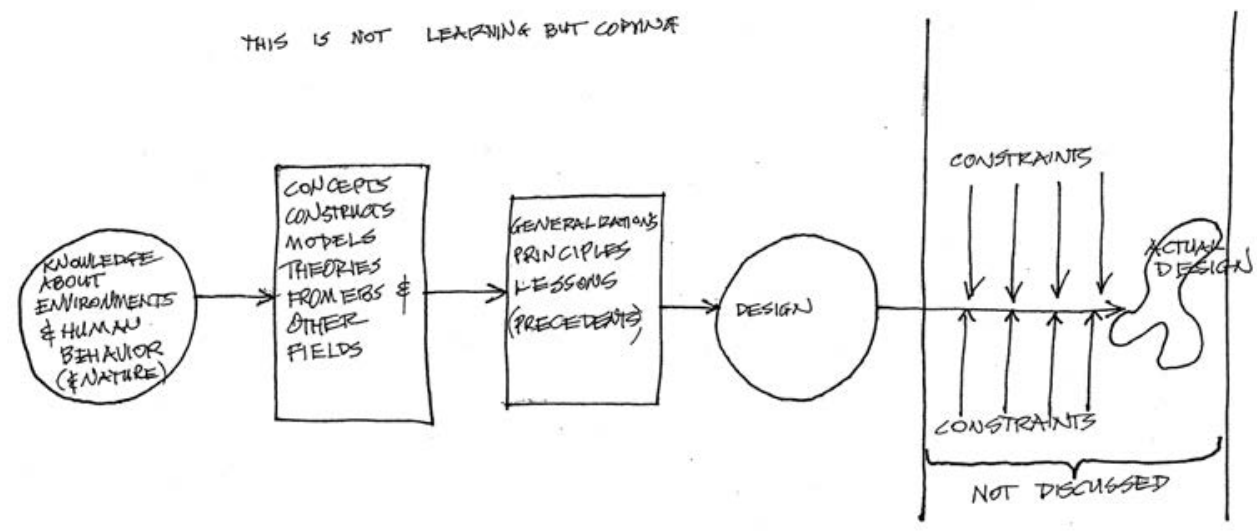

\section{CONSTANCIA}

Cambio, novedad y diferencias, entre otros tienden a ser enfatizados. Observar la constancia podría proporcionar un correctivo útil.

Mi énfasis está en la investigación (sobre todo en EBS visto en términos generales y como una disciplina científica). La investigación es fundamental en la ciencia y la investigación

4 RAPOPORT, A. "On the attributes of tradition". In J-P. Bourdier and N. Aesayyad (eds.) Dwellings, settlements and tradition, Lanham, MD. University Press of America. 1989. Pp. 77-I05 + RAPOPORT, A. History and precedent in Environmental Design, New York. Plenum, 1990. + RAPOPORT, A. "Levels of meaning and types of environments". In Y. YOSHITAKE et.al. (Eds.) Current Issues in Environment-Behavior Research. Tokio, University of Tokio. 1990. Pp. I35-148

5 RAPOPORT, A. "Defining vernacular DESIGN". In: TURAN, M. (Ed.) Vernacular architecture (Paradigms of Environmental Response. Aldershot. (UK). Avebury. 1990. pp. 67-101.

6 RAPOPORT, A. "On cultural landscapes”. In: Traditional Dwellings and Settlements Review. Vol. 3, No. 2. (Spring). 1992. PP. 33-47.

7 RAPOPORT, A. “Development, culture change and supportive design”. In: Habitat International. Vol. 7 No. 5/6. 1983. pp. 249-268. + RAPOPORT, A. Sustainability, Meaning and Traditional Environments. Berkeley, CA. Center for Design Research. University of California. Traditional Dwellings and Settlements Working Paper Series. Vol. 75. 1994. (IASTE 75-94)

8 RAPOPORT, A."Environmental quality and environmental quality profiles". In: RAPOPORT, A. Thirty-Three Papers in Environment-Behavior Research. Newcastle (UK). Urban International Press. 1995 [1990]. pp. 489-5I2. + RAPOPORT, A. Culture, Architecture and Design. Chicago. Locke Scientific. 2005.

9 RAPOPORT, A. "Vernacular design as a model system". In: L. Asquith and M. Vellinga (Eds.). Vernacular Architecture in the 2 I st Century: Theory, Education and Practice. Abingdon, Oxon (UK) Taylor \& Francis. 2006. Pp. I79-I98.

10 RAPOPORT, A. "Learning about settlements and energy from historical precedents". In: Ekistics. Vol. 54, No. 325-327 (July-Dec). 1987. pp. 26I-268. + RAPOPORT, A. "Settlements and energy: historical precedents" In: RAPOPORT, A. ThirtyThree Papers in Environment-Behavior Research. Newcastle (UK). Urban International Press. 1995. pp. 437-456
Figura I. Aprendizaje desde el amplio campo de los ambientes \& conocimiento. Fuente: Imagen suministrada por el autor. 
en curso es crucial. Proporciona el conocimiento cada vez mayor que puede conducir a diseños basados en la evidencia'". Dicha investigación debe incluir el estudio del pasado.

\section{CONSTANCIA CON RESPECTO A LAS PERSONAS}

Hay un grupo grande y creciente de trabajo en una serie de campos, que hace hincapié en la constancia respecto a las personas, basadas en su evolución. Este trabajo es la ecología del comportamiento, la socio-biología, la psicología evolutiva, la primatología, e incluso los estudios en animales sugieren que hay universales humanos, la naturaleza humana ${ }^{12}$. Estos trabajos han incluso comenzado a influir en la antropología (aunque muy lentamente) - el ejemplo es una nueva revista de antropología y naturaleza humana.

Este trabajo sugiere que la cultura no es totalmente variable, pero tiene constancias importantes. Por otra parte, implica que los aspectos aparentemente variables pueden ser diferentes expresiones de las constantes universales o compartidas por todas las culturas ${ }^{13}$.

Esta visión ha sido reforzada por el trabajo de Cronk ${ }^{14}$ que utiliza el concepto de "hiperespacio etnográfico", en el que se puede visualizar un gran número de posibles (imaginables) culturas, sobre la base de combinaciones de un número limitado de componentes aceptados de las culturas (ej. De los campos de Relaciones Humanas de la zona). Se estima que este número es 12 seguido por 52 ceros, de hecho, por supuesto, el número de cultivos humanos es mucho menor. Hauser ${ }^{15}$ hace la misma observación acerca de la cultura y una de sus importantes expresiones, el lenguaje.

Recientemente se ha descubierto ${ }^{16}$ que las redes sociales entre los cazadores-recolectores (los Hadza, en Tanzania), representantes de los primeros seres humanos, son las mismas que las redes sociales modernizadas actuales. Muestran notable constancia, especialmente teniendo en cuenta los cambios en la escala de la sociedad, tecnología de comunicaciones y nuevas instituciones sociales, entre otras. Por otra parte, las variables explicativas son también las mismas: "Estética" en general ${ }^{17}$ y, aún más escéptico soy sobre su valor en el diseño medioambiental el cual veo como una profesión basada en la ciencia, no un $\operatorname{arte}^{18}$. Sin embargo, es importante el hecho de que hay un creciente énfasis que parece ser válido en la universalidad de la estética, incluso en el hecho de la preferencia estética transculturalmente como un aspecto de la naturaleza humana. Esto se expresa en formas

II RAPOPORT, A. “Designing for people: some implications” En: TRIALOG, No. 106. 3, 20I0. pp. 4-45.

12 Por ejemplo, ver BROWN, D.E., Human Universals, Philadelphia, Temple University Press. 1991. Para más detalles y muchas más referencias, ver además RAPOPORT, A. "Designing for people: some implications".

I3 BROWN, D.E. Human Universals. Philadelphia: Temple University Press, I99| + GOLDSMITH, T.H., The biological roots of human Behavior. New York: Oxford University Press, I991 + PINKER, S. The blank slate (the modern denial of human nature). New York: Viking, 2002 + RHOADS, S.E. Taking sex differences seriously. San Francisco: Encounter Books, 2004 + McINTYRE, L. Dark ages (the case for a science of human behavior). Cambridge, MA.: Bradford Books/MIT Press, 2006 + CARTWRIGHT, J. Evolution and human behavior. London: Palgrave, 200I + ALCOCK, J. The triumph of sociobiology. Oxford: Oxford University Press, 200 I + BETZIG, L. (Ed.). Human nature: a critical reader. New York: Oxford University Press, 1997 + KONNER, M. The evolution of childhood. Cambridge, MA: Belknap Press of Harvard University, $2010+$ BARKOW, J.W., et.al. The adapted mind (evolutionary psychology and the generation of culture. New York: Oxford University Press, 1992, entre muchos otros.

14 CRONK, L. That complex whole (culture and the evolution of human behavior). Boulder CO:Westview Press, 1999.

I5 HAUSER, M.D. “The possibility of imposible cultures". In Nature, Vol. 460, Issue 7252, July. 2009. pp. $90-196$.

16 APICELLA, C.L, et.al. "Social networks and cooperation in hunter-gatherers". In Nature, Vol. 48I, Issue 7382, January. 20I2. pp. 497-50I.

17 RAPOPORT, A. The meaning of the build environment. Tucson: University of Arizona Press, 1990.

18 En lugar de hablar de estética es más útil pensar en los aspectos educativos, perceptivos, afectivos y asociacionales de la calidad del medio ambiente, ver Rapoport, 2010 y referencias en él. 
específicas en las culturas ${ }^{19}$. Una opinión similar se ha expresado acerca de la religión ${ }^{20}$. Esto sin duda se aplica a muchos otros aspectos de la cultura.

Los desarrollos ofrecen un importante correctivo para mucho del trabajo en la cultura y el medio ambiente ( $y$ las ciencias sociales en general), los cuales, al enfatizar la cultura $y$ descuidar la naturaleza humana se concentran sólo en la variabilidad; Este correctivo tiene implicaciones prácticas. Se puede reducir la variabilidad necesaria para considerar y tratar los numerosos estilos de vida (que representa a las culturas) cuando se trata del diseño', puede sugerir regularidades subyacentes y también ayudar a la transferibilidad de investigación solo de una cultura a otras ${ }^{22}$.

\section{CONSTANCIA EN LOS ENTORNOS}

Puesto que voy a discutir un ejemplo (barrios) con cierto detalle más adelante, esta sección será breve. Hay viviendas aparentemente diferentes, muchísimas en todo el mundo, he contado I.278 distintos ejemplos en la enciclopedia de arquitectura vernácula y no es una cobertura completa ${ }^{23}$, sin embargo, si se considera la forma más que la figura ${ }^{24}$, entonces se configura la organización del espacio y dominios, y el comportamiento del cual todos los ambientes están compuestos ${ }^{25}$. También he mirado y fotografiado los mercados (especialmente los mercados al aire libre) durante décadas y he hallado que su organización, espacios, y demás, muestra mucha constancia (ver http: www.sadp.ku.edu/Rapoport). Este se aplica claramente al espacio de los peatones. Esto puede aplicarse incluso a las ciudades del futuro, que no pueden ser tan diferentes - el número de posibles paisajes culturales (ciudades que han sido transformadas por el hombre), que son la unidad más útil de análisis, es limitada al igual que las culturas ${ }^{26}$.

La constancia es más evidente a escalas pequeñas, en el caso de los asentamientos humanos a escala de barrios, en lugar de ciudades, metrópolis, y regiones urbanas, entre otros. ${ }^{27}$. A esa escala más pequeña la constancia es notable.

19 DUTTON, D. "The art instinct". Beauty, pleasure and human evolution. New York: Bloomsbury Press, 2010 + MILLER, G. The mating mind. New York: Doubleday, $2000+$ SOLSO, R. L. The psychology of art and the evolution of the conscious brain. Cambridge, MA: MIT Press, 2003 + ZEKI, S. Inner vision (an exploration of art and the brain). Oxford: Oxford University Press, 1999.

20 WADE, N. The faith instinct, New York: Penguin Books, 2010+ LEWIS-WILLIAMS, D. Conceiving God .The cognitive origins and evolution of religion. London: Thames \& Hudson, 2010.

2I RAPOPORT, A."On the size of cultural groups". In Open house international, Vol. 27, No. 3, September, 2002. pp. 7-II + RAPOPORT, A. Culture, architecture and Design. Chicago: Locke Scientific, 2005 + RAPOPORT, A. "Some further thoghts on cultura and environment". In International Journal of Architectural Research. Vol. 2, Issue I, March. 2008. pp. 16-39.

22 RAPOPORT, A."Environment-behavior research in an Asian-Pacific context". In Journal of Asian Urban Studies. Vol.3, No, 3. March. 2002. pp. 17-20 + RAPOPORT, A."Environment-behavior studies: Past, present and future". In Journal of Architectural and Planning Research. Vol. 25, No. 4. Winter. 2008. Pp. 276-28I + RAPOPORT, A."Mind, land and society in an environment-behaviour perspective". In Architectonics I5. Barcelona: Ediciones UPC. 2008. pp. 33-61. Además, veáse Rapoport, A., Culture, architecture and Design. Figs. 37, 38 y 39, p. 80-8I.

23 RAPOPORT, A." Vernacular design as a model system". In ASQUIT, L. and VELLINGA M. (Eds.) Vernacular achitecture in the 2 I st century: Theory, education and practice. Abingdon, Oxon (UK): Taylor and Francis, 2006. pp. 179-180.

24 RAPOPORT, A. Human aspects of urban form, Oxford: Pergamon Press, 1977.

25 RAPOPORT, A. "Systems of activities and systems of settings". In: KENT, S. (Ed.) Domestic architecture and the use of space. Cambridge: Cambridge University Press, 1990. pp. 9-20 + RAPOPORT, A."Space organization and the built environment". In: INGOLD, T. (Ed.) Companion enciclopedia of antropology. London: Routtedge, 1994. pp. 460-502 + RAPOPORT, A. Culture, architecture and design. Chicago: Locke Scientific, 2005.

26 A mediados de la década de los 60, cuando estaba en el Bartlett, un estudiante analizó las ciudades como se ven en ciencia - ficción. El encontró que había, por ejemplo, muchas de las tecnologías extraordinarias descritas, pero todas las ciudades eran como Ville Radiance de Le Corbusier o Ciudad Broadacre de Frank Lloyd Wright. (Véase RAPOPORT, A. The meaning of the built environment. Tucson: University of Arizona Press, 1990.)

27 RAPOPORT, A. Human aspects of urban form, Oxford: Pergamon Press, 1977. + RAPOPORT, A."The city of tomorrow, the problems of today and the lessons of the past". In: RAPOPORT, A. Thirty three papers in Environment-Behaviour Research". Newcastle (UK): Urban International Press, 1995 [1973]. pp. 189-200. + RAPOPORT, A. "The nature and role of neighborhoods". In: SHOKOOHY, M. (Ed.) Urban Design Studies. Vol 3. London: School of architecture and landscape, University of Greenwich, 1997. pp. 93-118. + RAPOPORT, A. RAPOPORT, A."On the size of cultural groups". In Open house international, Vol. 27, No. 3, September, 2002. + RAPOPORT, A. ."Vernacular design as a model system". In ASQUIT, L. and VELLINGA M. (Eds.) Vernacular achitecture in the 2 Ist century:Theory, education and practice. Abingdon, Oxon (UK): Taylor and Francis, 2006. 
A gran escala la ciudad contemporánea es diferente a las del pasado. La metrópoli se ha descrito como la nueva forma, por primera vez, en 2000 años, al igual que las regiones urbanas y megalópolis. Pero como he sostenido desde hace algún tiempo la gente no vive en estos, sino que son conceptos éticos y construcciones. Las personas todavía viven en áreas relativamente pequeñas (a veces incluso, cada vez, más pequeñas, aunque la forma como viven en ellas y las usan puede variar en los diferentes grupos.

Puesto que los barrios son pequeños y, a menudo, homogéneos en lo que respecta a varias dimensiones, (etnia, raza, tribu, ocupación, religión, nivel económico, y sobre todo, estilo de vida), son numerosos. Ya he citado algunas cifras ${ }^{28}$. Viajes recientes apoyan esto. Por ejemplo, en 2009 visité el centro de Asia y encontré que las áreas tradicionales de Tashkent, Bujara y otros se componen de muchos barrios pequeños. Las nuevas áreas no parecen tener esto y me permito sugerir que la planificación y el diseño contemporáneos no tienen en cuenta este importante aspecto de los asentamientos.

Estas pequeñas unidades pueden estar relacionadas con lo que el sociólogo llama instituciones intermedias o estructuras mediadoras, que como se verá más adelante, juegan un gran papel. En nuestro caso estas son unidades intermedias socio-espaciales entre el individuo o la familia y la más grande es la social o urbana y es la parte más importante del sistema de vivienda-asentamiento ${ }^{29}$.

Esta fuerte tendencia también es confirmada por la investigación en curso. Por ejemplo, la parte tradicional de Kirtipur, un pequeño pueblo en Nepal, tiene más de 30 barrios homogéneos que faltan en las nuevas áreas ${ }^{30}$. La ciudad de Milwaukee tiene 186 barrios definidos que, como ya predije, se subdividen ${ }^{31}$. Esto parece estar ocurriendo a juzgar por los nuevos nombres que aparecen en las señales correspondientes a las que pone la ciudad. Por otra parte, los barrios étnicos se han desarrollado mientras la inmigración continúa ${ }^{32}$.

Considere un ejemplo en concordancia con mi argumento general sobre el valor de la utilización de novelas, revistas, periódicos, anuncios y otros ${ }^{33}$. En una memoria Epstein señala que Chicago tiene 237 barrios $^{34}$, (yo creo que bastantes más, y se subdividen como en Milwaukee). A través de la vivienda es posible predecir el origen étnico, ingresos, comportamiento, uso del espacio e instituciones. Cada barrio es efectivamente un pueblo, que se define por la pertenencia étnica, tradiciones, costumbres y demás que la gente realmente dejó en su barrio, todo lo que necesitaban estaba en ellos. [Esto tiene cambios para muchos, pero no todos los grupos ${ }^{35}$. Además, mientras cambian los barrios, puede que de nuevo lleguen a ser homogéneos, como lo muestran los distintos grupos de estilo de vida que pueden ser identificados por los códigos postales en los EE.UU ${ }^{36}$. Por otra parte, las diferencias entre los residentes restantes y los que van llegando pueden dar lugar

28 RAPOPORT, A. Human aspects of urban form, Oxford: Pergamon Press, 1977. pp. 250-255.

29 Ibíd., + RAPOPORT, A. "Systems of activities and systems of settings". In: KENT, S. (Ed.) Domestic architecture and the use of space. Cambridge: Cambridge University Press, 1990. + RAPOPORT, A."Space organization and the built environment". In: INGOLD, T. (Ed.) Companion enciclopedia of antropology. London: Routtedge, 1994.

30 SHRESTA, U. S., et. al. "Social effects of land use change in Kirtirpur, Nepal". In: SHOKOOHY, M. (Ed.) Urban design studies. Vol. 3. London: School of architecture and landscape. University of Greeenwich, 1997. pp. 5I-73.

3I RAPOPORT, A. Human aspects of urban form, Oxford: Pergamon Press, 1977.

32 DEARBORN, L.M., "Reconstituting among cultura and traditions in Milwaukee, Wisconsin". In: Traditional dwellings and settlements review. Vol. 19, No. 2. Spring, 2008. pp. 37-49

33 RAPOPORT, A."Indirect approaches to environment-behavior research". In: The National Geographical Journal of India. Vol. 36 (parts I and 2) March/June, 1990. pp. 30-46.

34 EPSTEIN, J. “Toddling town”. In: Commentary. Vol. I28, No. 4. November 2009. pp. 31-35. Ver además ejemplos adicionales en: RAPOPORT, A. “Designing for people: some implications” En: TRIALOG, No. 106. 3, 2010.

35 RAPOPORT, A. Human aspects of urban form,Oxford: Pergamon Press, 1977. Cap. 5.

36 RAPOPORT, A. The meaning of the build environment. Tucson: University of Arizona Press, 1990. + RAPOPORT, A. "Using 'culture' in housing design". In: Housing and Society. Vol. 25, No. I. (Outsummer 1999). 1998. pp. I-20. + RAPOPORT, A. "Theory,culture and housing". In: Housing, Theory and Society. Vol. 17, No. 4, 2000. pp. I45-I65. 
a nuevas subdivisiones ${ }^{37}$. Esto también establece nuevos tipos de homogeneidad - si los principios todavía aplican.

Esta tendencia también se observa a través del tiempo, según lo revelado por los viajes, la historia y la arqueología. En la actualidad existe mucho trabajo en la arqueología que lo demuestra, lo que refleja un creciente interés en estas cuestiones. Me limitaré a citar un ejemplo ${ }^{38}$. También he tenido trabajos de estudiantes que examinaron algunos de los estudios arqueológicos y mostraron la persistencia de algunos de los barrios. Una revisión sistemática de la literatura arqueológica pertinente sería muy útili39.

A pequeña escala se encuentra la constancia tanto en el tiempo como en el espacio lo que sugiere que la presencia de estas unidades es muy importante. Su naturaleza básica no ha cambiado (aunque son menos autónomas). Siguen presentes, su tamaño, es, en general, similar debido a la definición subjetivo-cognitiva como esquemas socio-espaciales ${ }^{40}$, tienden a ser homogéneos, así varíen los criterios de homogeneidad entre culturas. A pesar de que se producen cambios, estos cambios son mucho más lentos y diferentes a los de escalas más grandes (ciudad, metrópoli y región urbana, entre otros.). Estas constancias persistentes, tanto de las personas como de los entornos han sido posibles aprendiendo del pasado. (Como siempre en el nivel adecuado de abstracción).

Ocurre asimismo que, a mayor escala, los determinantes importantes tienden a ser la economía, la tecnología (de tráfico), la política y otras, mientras que a pequeña escala los aspectos humanos pueden llegar a ser dominantes. También en el tratamiento de la calidad del entorno de los asentamientos humanos, habrá, además de los usuarios, muchos otros actores implicados: políticos, empresarios, economistas, ingenieros, gerentes, urbanistas y diseñadores medioambientales (arquitectos, urbanistas, arquitectos paisajistas). Cabe señalar que algunos aspectos de la calidad del entorno no pueden ser cambiados por cualquiera de los actores, ej. el clima, la topografía, las vistas, la población y la composición social entre otras.

Otros componentes pueden ser cambiados por los políticos (nacional, regional y local), gerentes, empresarios y economistas, pero no por planificadores ni diseñadores, por ejemplo, puestos de trabajo y la economía, los impuestos, la calidad de la escuela, servicios sociales y urbanos (limpieza viaria, mantenimiento, recolección de basura, calidad del agua y demás.) Sin embargo, los planificadores y los ingenieros pueden influir, pero no los diseñadores (por ejemplo, el transporte público, grandes infraestructuras, la ubicación de varios usuarios y actividades, los sistemas de espacios abiertos, los marcos generales y otros), otros dependen de los usuarios- grupos e individuos. Por último, hay cosas que los diseñadores pueden hacer, y es, a pequeña escala, que "el diseño" comienza a jugar un papel y puede, en principio, enfatizar los deseos y necesidades de los usuarios.

Es útil identificar lo que distingue a los diseñadores de los otros actores y lo que los diseñadores medio ambientales tienen en común. Su dominio es de mediana a pequeña escala. Aquí se superponen con usuarios y tiene que tomar la cultura (grupo) en cuenta y maximizar

37 SUCHAR, C.S. and ROTENBERG. "Judging the adequacy of shelter: a case from Lincoln Park". Paper presented at the meeting for applied antropology. Tampa, Florida. (Mimeo), 1988.

38 SMITH, M.L. (Ed.) The social construction of ancient cities.Washington, D.C.: Smithsonian Books, 2003.

39 Téngase en cuenta que la arqueología es una de las "nuevas" disciplinas que EBS necesita considerar. Es significativo que un número de arqueólogos se han referido a mi trabajo, y se han involucrado con la arqueología

40 RAPOPORT, A. Human aspects of urban form,Oxford: Pergamon Press, 1977. + RAPOPORT, A. "The nature and role of neighborhoods". In: SHOKOOHY, M. (Ed.) Urban Design Studies. Vol. 3. London: School of Architecture and Landscape. University of Greenwich, 1997. pp. 93-I18. + RAPOPORT, A. "The role of neighborhoods in the succes of cities". In: Ekistics. Vol. 6, No. 4I5-4I7. July-Dec, 2002. pp. I45-I57. 
finales abiertos. Pero hay un aspecto que los que estudian las ciudades rara vez tienen en cuenta: lo tridimensional (i.e. espacial) y las cualidades experimentales de los entornos urbanos, a pesar de que están en todas las modalidades sensoriales (no sólo visual), son dinámicas, estáticas y demás, se trata de la percepción de los usuarios, los conocimientos y las evaluaciones que deben ser descubiertos. Además, aunque la organización espacial es claramente necesaria, no es suficiente. Por ejemplo, la configuración se debe considerar en relación con las actividades y el comportamiento. Además, el ambiente, la esencia de la experiencia del medio ambiente depende de los elementos semi-fijos y no fijos en el marco de los elementos de función fija ${ }^{41}$.

\section{LA NATURALEZA DE “DISEÑO”}

Pongo diseño entre comillas porque tomo una visión muy diferente de diseño de la que sólo voy a hablar muy brevemente ${ }^{42}$, en esta visión el diseño debe ser visto como basado en las pruebas de la investigación, no como arte, sino una profesión basada en la ciencia. El diseño tiene que verse como hipótesis, en base a la mejor investigación disponible, y ponerlas a prueba, ya sea por la simulación antes de su ejecución o la evaluación posterior a la ocupación después de la implementación y el resultado de la retroalimentación en nuevas bases de conocimientos.

En este sentido, además del método de investigación formal (básica, translacional, aplicado y evaluado) está el método "informal", que hace tiempo vengo resaltando la necesidad de usarlo: mediante el uso del dominio, como se describió anteriormente. El uso y la síntesis del conocimiento existente, incluido revisar trabajos anteriores y reinterpretarlos a la luz de nuevos conocimientos, utilizar lo que yo llamo métodos indirectos, el análisis de fuentes no profesionales brevemente mencionadas anteriormente ${ }^{43}$. También es útil para estudiar entornos, ver cómo se siente estar ellos. Esto incluye los entornos locales y remotos cuando uno camina, conduce a través de ellos o vuela sobre ellos. Esto depende de la observación, análisis y preguntas basadas en conocimientos previos, incluidas las actividades y el comportamiento en estos ambientes semi-fijos, los elementos y los cambios en el entorno, su naturaleza, las tasas de cambio, incluida la modernización en los países en desarrollo, y los efectos de la migración, tanto en países desarrollados como en vía de desarrollo (rural / urbano e internacional), por ejemplo, en la formación de enclaves étnicos y de otra índole.

Puesto que la inmigración es estresante, y se ha demostrado que tiene efectos sobre la salud tanto mental como física, modular rutas de cambio se vuelve importante ${ }^{44}$. En este caso, las pequeñas unidades urbanas que permiten la agrupación, homogeneidad, instituciones concretas, elementos semifijos, desempeñan un papel importante. Se convierten en apoyo para estos numerosos grupos, permiten la estructuración defensiva y también aumentar la variedad de ambientes. Esto, a su vez, aumenta la elección y selección del hábitat (que también aumenta la complejidad perceptiva y la riqueza de las ciudades).

$4 I \quad$ RAPOPORT, A. "On regions and regionalism". In: MARKOVICH, N.C., PREISER, W.F.E, and STURM, F.G. (Eds.) Pueblo style and regional architecture. New York: Van Nostrand Reinhold, 1992. pp. 272-294 (paperback edition only) + RAPOPORT, A. Cross-cultural studies and urban form.(The 1992 Lefrak Lectures), College Park, MD, University of Maryland, Urban Studies and Planning Program. 1993 + RAPOPORT, A. "On the perception of urban landscapes". In: Urban Design Studies. Vol. 6. London: School of Architecture and Landscape. University of Greenwich, 2000. pp. 129- 148.

42 RAPOPORT, A. "Environmental quality and environmental quality profiles". In: RAPOPORT, A. Thirty-Three Papers in Environment-Behavior Research. Newcastle (UK). Urban International Press. 1995 + RAPOPORT, A. Culture, Architecture and Design. Chicago. Locke Scientific. 2005. Para una discusión más amplia ver: RAPOPORT, A. “Designing for people: some implications" En: TRIALOG, No. 106. 3, 2010.

43 RAPOPORT, A."Indirect approaches to environment-behavior research". In: The National Geographical Journal of India. Vol. 36 (parts I and 2) March/June, 1990.

44 RAPOPORT, A. "Development, culture change and supportive design". In: Habitat International. Vol. 7 No. 5/6. 1983. 
Hay un tema específico de los países en desarrollo que cobra importancia, ya que la mayor parte del crecimiento urbano futuro tendrá lugar en ellos. En 1983 señalé en primer lugar el conflicto entre el deseo de modernización y la necesidad de entornos tradicionales, familiares, específicamente culturales, orientados hacia las necesidades de los humanos y con finales abiertos. Hay mucha evidencia que ha hecho hincapié en esta cuestión, que también tiene un impacto en la "sostenibilidad" 45 y conservación ${ }^{46}$. Cómo conciliar ambas se convierte en un tema importante del que hablaré más adelante, en términos de la matriz / infiltración ${ }^{47}$.

\section{El papel de nuevas (y "poco probables") disciplinas - un ejemplo}

Me he referido brevemente al hecho de que si enfocados al mismo nivel de abstracción, muchos de los nuevos y a menudo "poco probables" campos se vuelven relevantes. He añadido desde hace algún tiempo muchos nuevos campos científicos, principalmente los pocos con los que comenzó EBS ${ }^{48}$.

Aquí se discute un ejemplo sobre un campo muy "improbable" que sobre el terreno resultó ser increíblemente importante para mi discusión anterior de la constancia de las personas. Este fue un artículo sobre la construcción nacional (sobre todo en relación con Afganistán, pero también en referencia a Irak y Pakistán ${ }^{49}$. El concepto a tratar es el uso de "abajo hacia arriba" ${ }^{50}$. Resumo seis puntos generales abstraídos del contexto específico de la construcción de la nación, que muestran cómo se relacionan con mi análisis anterior sobre las pequeñas unidades urbanas y cómo, juntos, dan lugar a sugerencias acerca de la planificación y el diseño de los asentamientos humanos.

I. No sólo las sociedades tradicionales, sino también las llamadas sociedades modernas de masas se componen de comunidades o grupos [que Etzioni llama "tribus"]. Los individuos son miembros de muchos grupos, étnicos, religiosos, tribales y lingüísticos, entre otros, y yo añadiría, grupos de sexo, edad, educación y estilo de vida). Estos no tienen que ser residenciales [a pesar de que pueden serlo, ya a menudo lo son]. Los miembros de estos grupos comparten reglas sobre qué hacer y no hacer, costumbres, creencias, rituales, redes sociales, resolución de conflictos, y demás. (Estos grupos corresponden a los muchos grupos de estilo de vida pequeños que yo había enfatizado previamente ${ }^{51}$.

45 RAPOPORT, A. Sustainability, Meaning and Traditional Environments. Berkeley, CA. Center for Design Research. University of California. Traditional Dwellings and Settlements Working Paper Series. Vol. 75. 1994

46 RAPOPORT, A. “Traditional environments, culture and preservation”. In: TURGUT, H. and KELLET, P. (Eds.) Traditional environments in a new millennium. Istanbul: Istan bul Technical University IAPS-CSBE. 2002. pp. 26-32.

47 RAPOPORT, A. "On cultural landscapes”. In: Traditional Dwellings and Settlements Review. Vol. 3, No. 2. (Spring). 1992.

48 RAPOPORT, A. Culture, Architecture and Design. Chicago. Locke Scientific. 2005 + RAPOPORT, A. "Environment-behavior studies: past, present and future”. In: Journal of architectural and planning research. Vol. 25, No. 4. Winter. 2008. pp. 27628 I + RAPOPORT, A. "Mind, land and society ina an environment-behavior perspective". In: Arquitectonics I5. Barcelona: Ediciones UPC. 2008. pp. 33-6I + RAPOPORT, A. "Some further thoughts on culture and environment". In: International Journal of Architectural Research. Vol. 2, Issue I. March, 2008. pp. 16-39 + RAPOPORT, A. Culture, Architecture and Design. Chicago. Locke Scientific. 2005. Para una discusión más amplia ver: RAPOPORT, A. “Designing for people: some implications" En: TRIALOG, No. 106. 3, 2010.

49 ETZIONI, E. “Bottom-up nation building”. In: Policy Review. No I58. December, 2009. pp. 5 I-62.

50 Nótese que este concepto puede utilizarse también en otras formas que no son tan útiles para mis propósitos. También se ha aplicado a la planificación; un ejemplo de ello es la participación del usuario (que es un tema diferente pero importante en la planificación y diseño ambiental). Nótese también que el método "indirecto" de investigación discutido anteriormente también puede ser visto como de abajo hacia arriba, en el sentido en que proporcionan las reacciones inconscientes de la gente, y la evaluación de los ambientes.

5I RAPOPORT, A. “Using 'culture' in housing design”. In: Housing and Society. Vol. 25, No. I. (Outsummer 1999). $1998+$ RAPOPORT, A. "Theory, culture and housing". In: Housing, Theory and Society. Vol. 17, No. 4, 2000 + RAPOPORT, A. "On the size of cultural groups". In Open house international, Vol. 27, No. 3, September, 2002 + RAPOPORT, A. Culture, Architecture and Design. Chicago. Locke Scientific. 2005 + RAPOPORT, A. "Some further thoughts on culture and environment". In: International Journal of Architectural Research. Vol. 2, Issue I. March 2008. 
2. La pertenencia a grupos influencia las decisiones individuales en cuanto a alimentación, vestido, comportamiento y demás. Por lo tanto, hay regularidades dentro de los grupos y no son "arbitrarias". Pueden estar representadas por el estilo de vida e incluyen decisiones ambientales ( $y$ el diseño como un proceso de elección) ${ }^{52}$.

3. Los lazos dentro de los grupos son más fuertes que entre otros grupos. Dentro de los grupos varias personas, líderes religiosos, curanderos, comerciantes, de élite y otros son muy influyentes. Estas fuerzas internas, o informales y la identidad de grupo son mucho más fuertes que las fuerzas más grandes o más formales (en este caso el gobierno nacional). Yo añadiría que la identidad de estos grupos es a menudo en contraste con la de los demás - "nosotros no somos como ellos". Esto también conduce a una estructuración defensiva con implicaciones para los entornos (incluyendo ciudades) como los organismos competentes de espacio, tiempo, significado y comunicación, elementos fijos y no fijos, sistemas de valores, paisajes culturales y su ambiente, clara comunicación no verbal.

4. De ello se desprende que uno tiene que trabajar con lo que existe en lugar de empezar desde cero, de novo, trabajar en contra de ello (o luchar contra ello), se debe trabajar con lo que existe. (Es significativo que Etzioni utilice el término "diseño" en conexión con las instituciones nacionales). Creo que este enfoque se aplica en gran medida a las constancias de los elementos de la pequeña escala en las ciudades, lo que he subrayado.

5. Al crear, no sólo se debe utilizar lo que existe, sino también lo que se quiere que sea. Es más fácil y productivo ir con la corriente, dirigirla y guiarla en lugar de buscar algo completamente nuevo que es desconocido, posiblemente impracticable e inaceptable, posiblemente mal y a menudo rechazado ${ }^{53}$.

6. En lugar de diseñar un enfoque o el resultado, primero debemos preguntarnos cuál es la realidad sobre el terreno, cuáles son los elementos o instituciones existentes, cómo y en qué dirección cambian y en el alcance [y la velocidad] de los cambios de diversos elementos. Y lo que podrían aportar ${ }^{54}$.

\section{Algunas consecuencias del diseño. Un ejemplo}

En "Aspectos humanos de la forma urbana", defendiendo la importancia de las pequeñas unidades urbanas homogéneas, en parte debido a su persistencia interculturalmente y con el tiempo, dibujé el siguiente diagrama ${ }^{55}$. (Ver figura 2)

52 RAPOPORT, A."On the size of cultural groups". In Open house international, Vol. 27, No. 3, September, 2002 + RAPOPORT, A. Culture, Architecture and Design. Chicago. Locke Scientific. 2005.

53 A menudo hay buenas razones para lo que existe, incluso en casos aparentemente sencillos. Estos deben ser entendidos en vez de ignorados y sustituidos. En la década de 1950 (no tengo la referencia) asesores agrícolas de las UN abogaron por la eliminación de los montones de piedras en los campos. Eso empeoró las cosas - las piedras estaban atrapadas en un ambiente árido. He discutido ejemplos comparables de tales consecuencias negativas no intencionales en entornos construidos (Rapoport 2005, pp. 2-9). También hay muchos casos documentados de denegación de nuevas viviendas, donde las personas han tenido que regresar a los asentamientos espontáneos. Si la vivienda se tolera los resultados podrían ser negativos.

54 RAPOPORT, A. "Development, culture change and supportive design". In: Habitat International. Vol. 7 No. 5/6. $1983+$ RAPOPORT, A. and HARDIE, G. "Cultural change analysis: core concpets of housing for the Tswana". In: TIPPLE, A. G. and WILLIS, K.G. (Eds.) Housing for the poor in the third world (Methods, analysis, case studies and policy). London: Routledge, 1991. pp. 35-61.

55 RAPOPORT, A. Human aspects of urban form, Oxford, Pergamon Press. 1977. (Fig. 5.4, p. 263) 


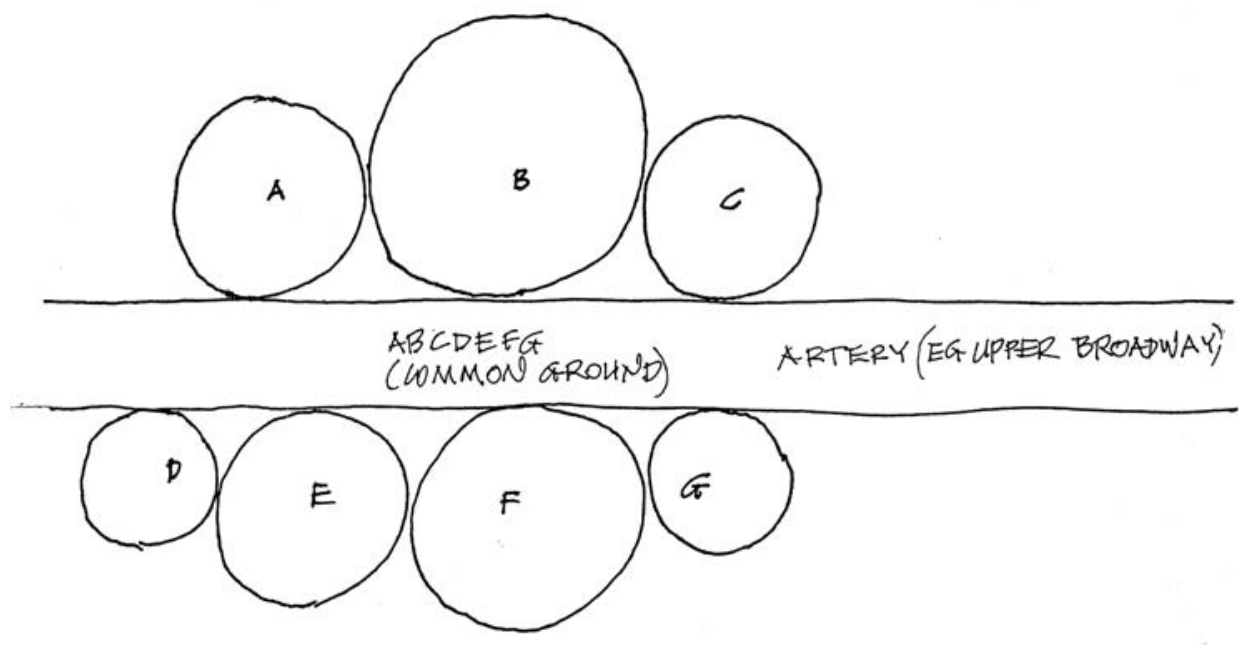

Este diagrama trata de ilustrar el área de la parte alta de Broadway, en Manhattan. Fue muy abstracto - Nueva York no es realmente así. La gente, sin embargo, trata de agruparse y definir los límites subjetivos de los barrios lo más claramente posible, y aunque el diseño puede ayudar, la definición no es tan clara o concreta como en el diagrama. Sin embargo, representa la realidad de muchos lugares. Durante largos viajes, fue puesto de manifiesto que, sobre todo en Asia, pero también en otros lugares, detrás de grandes avenidas, con edificios modernos de oficinas y hoteles, se encuentran pequeñas áreas totalmente tradicionales y bien definidas. Este es el caso de China, India, Tailandia, Indonesia, Asia Central, $y$ sin duda otros lugares ${ }^{56}$.

Generalización: estas observaciones pueden generalizarse y diagramarse de la siguiente manera. (ver figura 3)

Este modelo tiene muchas ventajas y tiene mucho que enseñarnos, si lo entendemos y derivamos lecciones de él ${ }^{57}$ (ver figura l). Brevemente se revisan algunas de estas ventajas.

I. El patrón ofrece un "frente" a lo largo de las arterias principales, el entorno global, moderno y caro, la imagen apropiada y la señalización importante costosa ${ }^{58}$. Estos pueden responder a la tecnología, la economía, la política y el tráfico, entre otros.

2. Este modelo también ofrece una "nueva" región - los espacios de tradición local, el entorno para las actividades tradicionales, vendedores, servicio de lavandería, cartas o juegos, el significado local, señales no verbales y las instituciones. Se trata de la cultura - específica y si de composición abierta, permite la personalización, tanto de los individuos como de los grupos. Puesto que pueden ser homogéneos, esta

56 RAPOPORT, A. "The nature and role of neighborhoods". In: SHOKOOHY, M. (Ed.) Urban Design Studies. Vol. 3. London: School of architecture and landscape, University of Greenwich, 1997 + RAPOPORT, A. "The role of neighborhoods in the success of cities". In: Ekistics. Vol. 6, No. 415-4I7. July-Dec, 2002 + RAPOPORT, A. "Local environments in a global context". In: Ekistics. Vol. 73, No. 436-44I. Jan-Dec. 2006.

57 Es importante recordar que a gran escala (ciudad, metrópoli, urbana, Región) las personas aún viven en unidades más pequeñas de las cuales consisten estos asentamientos

58 RAPOPORT, A. "Mind, land and society in an environment-behaviour perspective". In Architectonics I5. Barcelona: Ediciones UPC. 2008. La nueva capital de Turkmenistán (Ashjabad), en un ejemplo extremo, siendo todo el frente, la imagen y "señalización costosa". Resulta muy significativo que tan sólo a I hora de distancia se encuentre uno de los mayores mercados más tradicionales de Asia Central (Tolkuchka).
Figura 2. Abstracción de la relación "vecindario/arteria". Fuente: Imagen suministrada por el autor. 
Figura 3. Relación actual vecindario/ vía arteric como se encuentra en India, China, Tailandic e Indonesia entre otros. Fuente: Imagen suministrada por el autor.

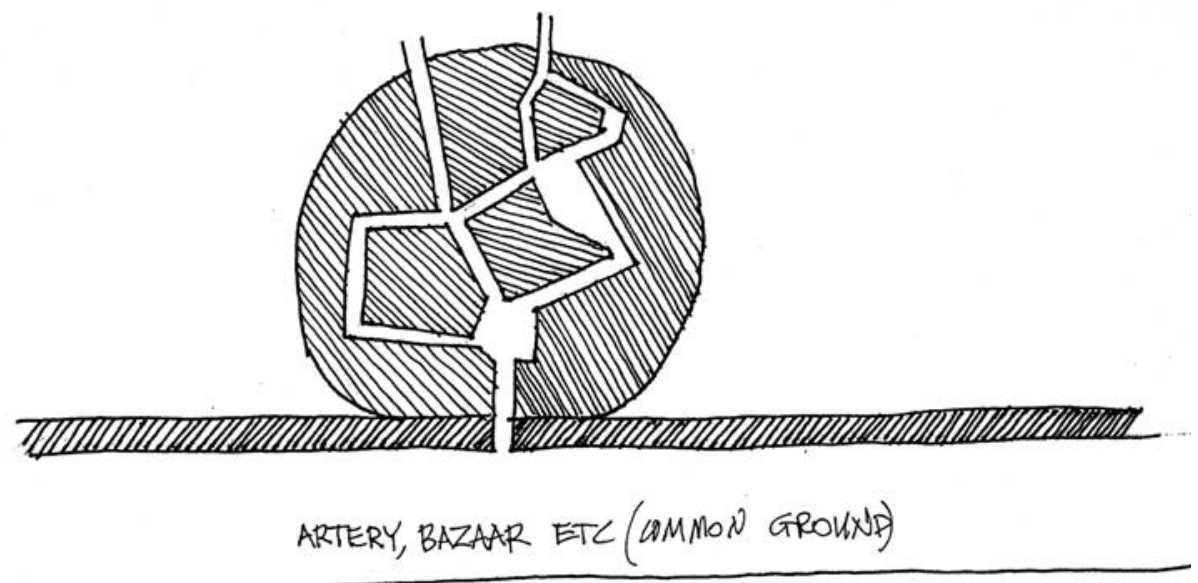

personalización se "suma”, y crea un ambiente específico ${ }^{59}$. Estas características son importantes en términos de toma de posesión, identidad, señales claras y fáciles de comunicación no verbal.

3. Las principales arterias constituyen "el espacio de las máquinas", las áreas traseras proporcionan espacios apropiados tanto para el comportamiento estático como dinámico de peatones y el tráfico lento. Cada uno de estos puede proporcionar las debidas cualidades perceptivas ${ }^{60}$.

4. La capacidad de estas áreas locales pequeñas para permitir la agrupación tiene una serie de consecuencias positivas. Localiza a las personas en el espacio social, es de apoyo para los diferentes grupos, permite la homogeneidad para maximizar la interacción, las normas locales y la comunicación no verbal, es sensible de muchas maneras, por ejemplo, puede permitir la operación de sistemas de reglas diferentes, y por consiguiente diseños del sitio diferentes y apropiados que pueden ser muy importantes culturalmente $^{61}$. Estas pequeñas unidades son el equivalente socio-espacial de las estructuras mediadoras / equivalente a las estructuras de mediación / mencionadas anteriormente.

5. Estas pequeñas áreas locales permiten la participación de finales abiertos, auto - construidos mediante sistemas de reglas diferentes y, por lo tanto, la creación de entornos adecuados con cambios continuos. Esto permite que la modernización tenga lugar para que lo que la gente quiere hacer no sólo está permitido, sino que se promueve

59 RAPOPORT, A. The meaning of the built environment. Tucson. University of Arizona Press. 1990. Ver: Fig. 21 , p. $138+$ RAPOPORT, A. "On regions and regionalism". In: MARKOVICH, N.C., PREISER, W.F.E, and STURM, F.G. (Eds.) Pueblo style and regional architecture. New York: Van Nostrand Reinhold, 1992.

60 RAPOPORT, A. Human aspects of urban form,Oxford: Pergamon Press, 1977. Cp. 4,esp, 240-247 + RAPOPORT, A. "The use and design of open spaces in urban neighborhoods". In: FRICK, D. (Ed.) The quality of urban life (social, psychological and physical conditions). Berlín: De Gruyter, 1986. pp. 159-I75 + RAPOPORT, A. History and precedent in environmental design. New York: Plenum, 1990. Parte III + RAPOPORT, A. "Pedestrian street use: culture and perception". In: VERNEZ MOUDON, A. (Ed.) Public streets for public use. New York: Columbia University Press, 1991. pp. 80-92

6I RAPOPORT, A. "Development, culture change and supportive design". In: Habitat International. Vol. 7, No. 5/6. 1983 + RAPOPORT, A. ."Environmental quality and environmental quality profiles". In: RAPOPORT, A. Thirty-Three Papers in Environment-Behavior Research. Newcastle (UK). Urban International Press. 1995. Nótese que no sólo se ignora la tendencia a agruparse, sino que puede que se prohíba la homogeneidad resultante, o al menos, se desaconseje fuertemente. Sin embargo, la gente sigue intentándolo (porque lo quieren). En Wisconsin, la propuesta de un pueblo Hmong cerca de Green Bay no se permitió, sin embargo, como hemos visto, los Hmong han tenido éxito en algunas agrupaciones en Milwaukee (Dearborn 2008) También he discutido un número de otros ejemplos recientes. 


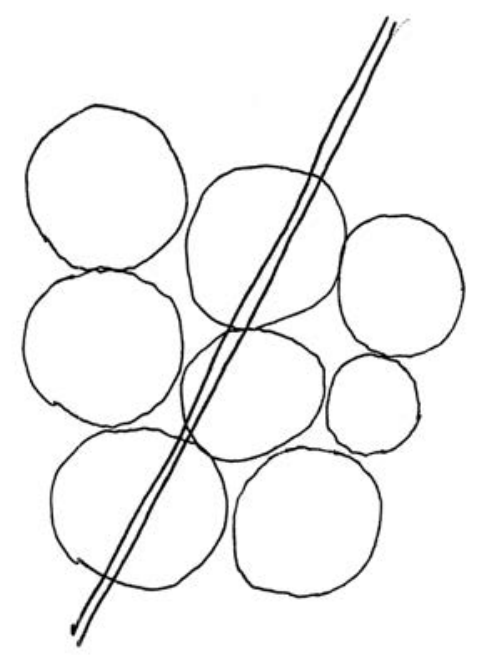

Figura 4. Alternativas a vías rápidas en un área urbana. Fuente: Imagen suministrada por el autor.

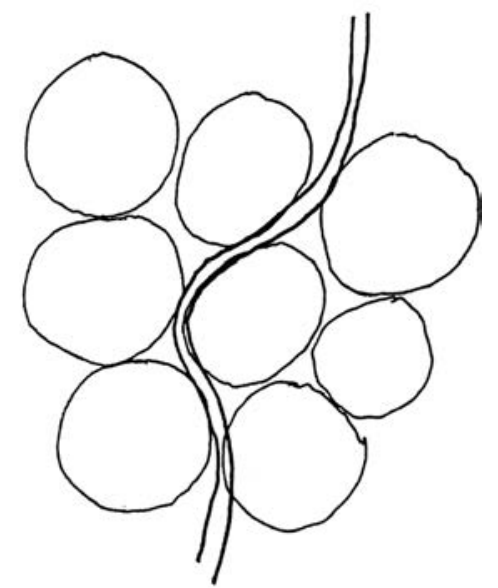

(lección de los asentamientos espontáneos). Los cambios pueden ocurrir dentro de los barrios de forma independiente y sin molestar a los sistemas urbanos más grandes, el marco y la imagen adecuada pueden permanecer sin cambios o pueden cambiar en respuesta a las distintas fuerzas ya mencionadas.

6. La presencia de pequeñas unidades claramente delimitadas también puede ayudar a las respuestas en esta escala grande. Por ejemplo, se hace más fácil "enhilar", por ejemplo, las carreteras entre barrios, no a través de ellos, mantienen su integridad y también vínculos importantes entre ellos. (Ver figura 4)

7. Dentro de las áreas pequeñas a menudo se encuentran Gradientes de Modernidad, el más moderno a la entrada y el menos moderno al "fondo". Esto permite una aculturación progresiva y una modernización permanente en curso. Los materiales modernos, servicios, y otros, pueden ser introducidos gradualmente para mejorar las condiciones materiales sin perder otras ventajas, por supuesto, esto sucede de forma natural en asentamientos espontáneos (por ejemplo, en América Latina) y puede ser dirigido como en el programa de mejora de Kamping en Indonesia. El proceso se puede continuar mientras el cambio cultural continúa, y aumenta la expectativa de la calidad del entorno ${ }^{62}$.

8. Dentro de estas áreas también se encuentran las gradientes de penetración con alguna medida de control sobre el territorio del grupo. Esto tiene implicaciones para el autocontrol y la seguridad.

9. Ya he mencionado que muchas de las características antes mencionadas reducen el estrés causado por la migración, el daño a la cultura y la organización. Estos procesos también han demostrado aumentar la incidencia de enfermedades mentales, por ejemplo, la esquizofrenia. Áreas homogéneas y responsive reducen este incidente. También mitigan los efectos de los entornos urbanos en general en los que la esquizofrenia es más frecuente que en el medio rural ${ }^{63}$.

62 SASTROSASMITA, S. and MURUL AMIN, A.T.M. "Housing needs of informal sector workers: the case of Yogyakarta, Indonesia". In: Habitat International. Vol. I4, No. 4. 1996.

63 VAN OS, J., et al. "The environment and schizophrenia". In: Nature, Vol. 468, Issue 732I November, 2010. pp. $203-2012$. Cabe señalar que en todos los casos la predisposición genética juega un papel. 
Figura 5. Resumen gráfico de lo que implica lo argumentado. Fuente: Imagen suministrada por el autor.
10. Las pequeñas unidades con las características descritas en los puntos I a 9 se acoplan a un ambiente específico, identidad y demás que puede cambiar en respuesta a cambios en la población común en todas las ciudades. Nuevos grupos pueden transformar las unidades, aumentando aún más la variedad. O’Donell ${ }^{64}$ estudió un área pequeña de la parte baja de Manhattan, a partir del siglo XVII. Mientras que las calles principales se mantuvieron sin cambios, hubo cambios importantes en la subdivisión de la tierra, actividades y circunstancias específicas de los sucesivos grupos diferentes que vivían allí. Más específicamente identificó un cambio reciente y en curso, desde "Little ltaly" hasta Chinatown, con cambios en los usos y el ambiente (a través de elementos semifijos, colores, olores, sonidos y actividades). Mediante el análisis de los cambios dentro de esa pequeña zona, O’Donnell sugirió la forma de elementos los finales abiertos.

I I. Además, estas pequeñas unidades discutidas en I a I I son importantes no sólo por permitir a los habitantes identificarse con las áreas, utilizarlos para la estructuración defensiva y demás. También crean una gran variedad de hábitats, con ambientes diferentes, diferentes organizaciones del espacio, tiempo, el significado y la comunicación, los colores, los sonidos, los olores, los elementos semi-fijos y demás. Esto hace la ciudad en su conjunto mucho más rica y más compleja a la percepción. También maximiza la elección entre los habitantes y la elección es un aspecto importante del efecto del ambiente sobre el comportamiento ${ }^{65}$. Por analogía, con la ecología se puede argumentar que la perseverancia de una gran variedad de hábitats (heterogeneidad del hábitat), que conserva la diversidad biológica también puede preservar la diversidad cultural, la pérdida de lo que puede ser como la pérdida de la bio y eco- diversidad. También se conserva una variedad de ambientes para su estudio y lecciones adicionales.

12. Los elementos anteriormente señalados, en I a 10, pueden ser parcialmente resumidos en un solo diagrama. (Ver Figura 5).

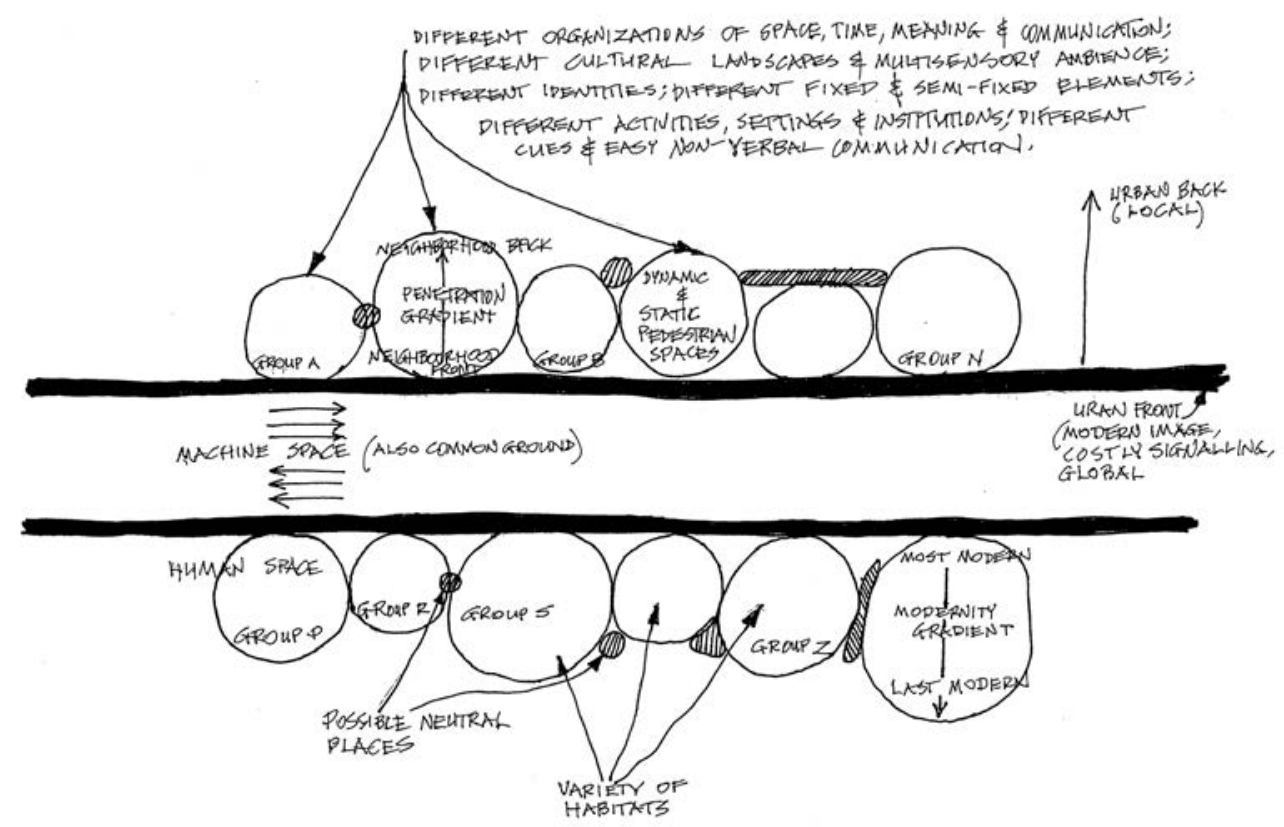

64 O'DONELL, S. Towards urban framework (accommodating change in urban cultural landscapes). Milwaukee, WI: Dept. of Architecture. M. Arch Thesis (unpublished)

65 RAPOPORT, A. Culture, Architecture and Design. Chicago. Locke Scientific. 2005. p. I I 


\section{CONCLUSIÓN}

Se puede entender muchas ciudades y paisajes culturales en general en términos de una matriz (a menudo de alto estilo) ${ }^{66}$ y esto puede estar relacionado con (1967) la vista de Bacon de las ciudades y la versión urbana del marco de Habraken ${ }^{67}$, lo que he descrito como espacio de las máquinas, ambientes que responden a fuerzas económicas, tecnológicas, políticas y de otra índole que forman la matriz. Las unidades pequeñas, he enfatizado y descrito, constituyen las construcciones de relleno. Hay un elemento de constancia incluso a mayor escala. Este enfoque, por lo tanto, saca provecho de lo que está ahí, lo que está sucediendo y lo que se "quiere" que pase. Esto hace que sea más fácil de implementar, dirigir, controlar y regular que imponer soluciones de cero que puede que ni siquiera funcionen.

Es aquí donde (2009) el enfoque de construcción de la nación de Etzioni y mi acercamiento a los asentamientos humanos se superponen. Los paralelismos son asombrosos. Conceptualmente, las conclusiones de estos dos ámbitos muy diferentes son muy similares y se refuerzan mutuamente. Este trabajo es casi como una versión física, a diferente escala y en un dominio diferente del argumento de Etzioni, que muestra el valor de usar una variedad de campos, si se aborda correctamente.

Considerado en términos generales, uno parece encontrar dos tendencias en conflicto. Por un lado, está el cambio global que aparentemente conduce a la difuminación de las distinciones y las reducciones de las diferencias, un paisaje urbano universal. Por otro lado, se encuentra un número creciente de grupos de estilo de vida, una búsqueda cada vez mayor de identidades culturales, étnicas y lingüísticas, regionales y locales. El conflicto entre estas dos tendencias posiblemente pueda ser resuelto por el método del marco. Los marcos pueden ser similares en todas partes para proyectar las imágenes deseadas. Pueden responder a todas las diferencias (incluida la aparente búsqueda de la imagen "sub-urbana") y con las ventajas descritas. Puede permitir sistemas de reglas diferentes, diferentes identidades, ofrecer espacios humanos y cualidades perceptivas adecuadas, pueden responder a la creciente repercusión de la migración - tanto rural como urbana e internacional.

El argumento de este trabajo es de un valor particular en los países en desarrollo, donde la mayor parte del crecimiento urbano se llevará a cabo. Pero también es relevante en los países desarrollados con el creciente número de grupos de estilo de vida, las opciones cada vez mayores y los límites más débiles, la migración internacional y la persistente y creciente importancia de los barrios persistencia.

Es, sin embargo, el estudio de las constancias en ambos, personas y ambientes lo que hace posible el aprendizaje a partir del pasado (y del presente) y la aplicación de esta lección para el futuro.

66 RAPOPORT, A. “On cultural landscapes”. In: Traditional Dwellings and Settlements Review. Vol. 3, No 2. (Spring). 1992. Fig. 5, p. 43.

67 RAPOPORT, A. "Flexibility, open-endedness and design". In: RAPOPORT, A. Thirty-Three Papers in Environment-Behavior Research. Newcastle (UK). Urban International Press. 1995.

M 19 REVISTA M VOL. 9 No.1. ENERO-JUNIO 2012 • FACULTAD DE ARQUITECTURA • UNIVERSIDAD SANTO TOMÁS COLOMBIA 


\section{APÉNDICE}

Completado el último borrador de estos documentos, algún material nuevo ha llegado a la mano. Refuerza los puntos importantes hechos en el documento. En primer lugar, la importancia de mantener la permanente investigación en diversos campos y la utilidad de los medios de comunicación populares. En segundo lugar, que una vez que los marcos conceptuales y teóricos son desarrollados, no solamente encajan los nuevos hallazgos, sino también es posible fácilmente "leer" los medios ambientes y mostrar cómo ambos encajan y refuerzan el argumento.

Sobre el primer punto, varias referencias útilmente pueden ser añadidas: Sigue esto. En cuanto al segundo describo una visita a un pueblo de Yunnan, China.

I. A. Cuatro (4) trabajos que muestran el uso de la constancia en diferentes escalas, en diferentes lugares y en relación con diferentes temas. (Abierto - Diseño terminado, la sostenibilidad, confort y apropiamiento cultural). Todos están en la publicación de Open House International, Vol. 36, No 4, 201 I.

(I) BAIGO, T. et al. "Future directions and sustainable buildings in Japan". Esto es basado en un análisis de casas tradicionales japonesas. p. 5-19.

(II) BEISI, J. y LINGYING, J. "Flexibility of traditional Buildings and craftsmanship in China" pp. 20-3I.

(III) NA, L.T.H. y PARK, J-H. "Applying eco-features of traditional Vietnamese houses to contemporary high - Rise housing”. Pp. 32-45.

(IV) AL-AMARECH, A. "The Bedouin tent in comparison with UAE housing provision". Estos usos y análisis de la tienda tradicional de invierno Beduino para evaluar la vivienda de interés social y sugerir mejoras relacionadas con los patrones de culturales y comportamientos. pp. 82-97.

I. B. Un libro aún no publicado en arqueología de la prensa de la Universidad de Arizona: ARNAULD, M.C., et al. (Eds) The Neighborhood as a social and spatial unit in Mesoamerican Cities. Esto hace eco y apoya mi trabajo sobre barrios y su importancia en general ${ }^{68}$ para el caso de Mesoamérica. Esto se basa en la disponibilidad de nuevas investigaciones en esa región y también relaciona el material a conclusiones similares en todo el mundo69. También de Calpulli en Tenochtitlán en: AGUILAR -. MORENO, M. Life in the Aztec World, Oxford University Press. 2006.

I. C. Newman, M. "Terraced living in a San Diego Quarry" New York Times, 2012. (29 de febrero). Esto describe los planes para una relativamente nueva y creciente ciudad estadounidense. El objetivo es convertir a San Diego en una "ciudad de aldeas" (De E.S. Rasmussens, temprano libro sobre Londres como una ciudad aldeas). De la descripción de estos pueblos se corresponden con las unidades que se ilustran en la Figura 5 en mis papeles. También hizo hincapié en la posibilidad como una orientación peatonal en estas

68 RAPOPORT, A. "The nature and role of neighborhoods". In: SHOKOOHY, M. (Ed.) Urban Design Studies. Vol. 3. London: School of architecture and landscape, University of Greenwich, 1997 + RAPOPORT, A. "The role of neighborhoods in the success of cities". In: Ekistics. Vol. 6, No 4I5-4I7. July-Dec. 2002.

69 SMITH, M.L. (Ed.) The social construction of ancient cities. Washington, D.C.: Smithsonian Books, 2003. 
unidades (de las figuras 3 y 5 del documento ${ }^{70}$ )

2. El 8 de abril de 2012, durante un viaje al suroeste de China para visitar los diversos grupos minoritarios, visité Xingwen aldea en la provincia de Yunnan. Este es un pueblo de Mongolia que ha estado allí desde los tiempos de Genghins Khan (siglo XIII), Ellos hablan tanto Mongol como Mandarín y usan ambas secuencias de comandos en las señales. Esto se muestra como persistente en los grupos étnicos en China y en otros lugares ${ }^{71}$. También de PAGEL, M. Wired for Culture, New York, W.W. Norton, 2012.

Físicamente, el pueblo es un ejemplo casi perfecto de las características de las unidades mostradas en la figura 5 del documento. (Ver análisis gráfico en Figura 6)

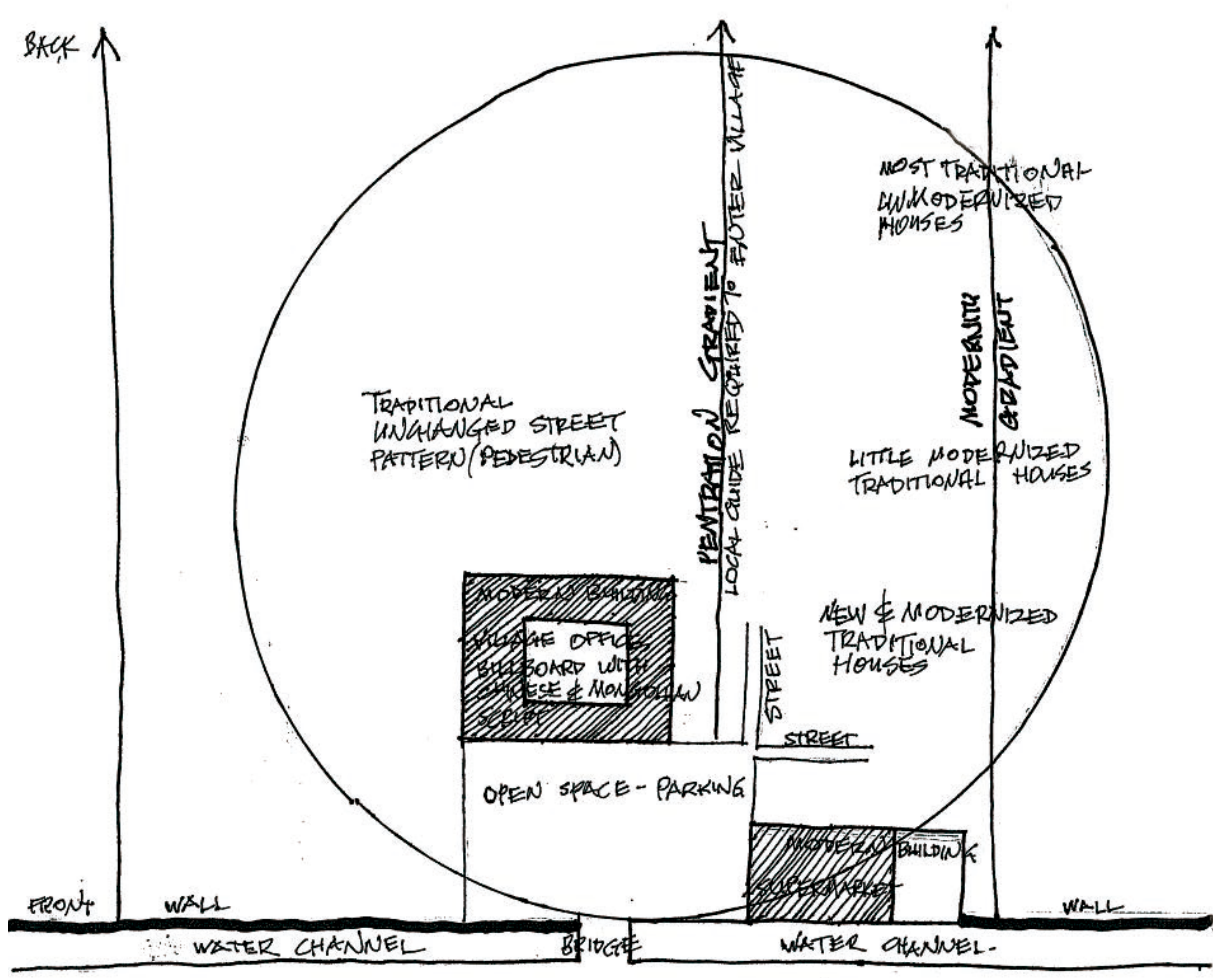

Figura 6. Xingmen Village. Confrontar con imágenes 3 y 5 en el documento. Fuente: Imagen suministrada por el autor

HIGHWAY

XINGMEN VILAGGE(YUNNAN,CHINA) NNAUSIS(CF.FIGS $3 \% 5$ IN PXPER)

Nota: Ver Bibliografía en p. 35 y subsiguientes

70 RAPOPORT, A. History and precedent in Environmental Design, New York. Plenum, 1990.

7I RAPOPORT, A. "Theory, culture and housing”. In: Housing, Theory and Society. Vol.I 7, No. 4. 2000 + RAPOPORT, A. "On the size of cultural groups". In Open house international, Vol. 27, No. 3, September, 2002 + DEARBORN, L.M., "Reconstituting among culture and traditions in Milwaukee, Wisconsin". In: Traditional dwellings and settlements review. Vol.19, No. 2. Spring. 2008. 


\section{THE ROLE OF CONSTANCY IN LEARNING FROM THE PAST. A LOST TRACK \\ Amos Rapoport \\ Universidad de winsconsin-Milwavkee, USA}

\section{INTRODUCTION}

There are several reasons for revisiting these topics

a. The themes addressed get stronger each time I revisit them, and receive support from new research findings, often from new disciplines'.

b. They increasingly come together and reinforce each other, esta blishing new linkages leading to generalization.

c. This, in turn, leads to very strong conceptual frameworks which play an important role in the development of (much needed) theory.

d. The new research findings not only strengthen the case being made, but suggest how learning can occur, and how one can benefit from ever new fields, using concepts and models from them, and ongoing research in them. This can only occur at some level of abstraction - any lessons are indirect.

The assumption that one can learn from the past presupposes some constancy, both regarding people and environment (settlements, dwellings, space, etc) as well as interactions between them ${ }^{2}$.

This constancy makes it possible to take advantage of the full range of built environments about which we know (and about which we are finding about more and more). As y have argued before, this involves a major expansion of the domain: including the whole environment all cultures, the full span of history (possibly including animal architecture) and the full range of environments including spontaneous settlements whish are not adeptly describe as "slums" and, if approached properly, have much to teach us (as vernacular trivial, popular and other environments) ${ }^{3}$. (Rapoport 1977, 1988, 1990 b,c)

This expansion of the domain is an incredible and still neglected resource, a record of human interactions with environments, their intentions, objectives and goals in creating environments, success and failures (and reasons for these), patterns and regularities processes, etc. The past represents an unparalleled laboratory if it is approached properly has a great deal to teach us.

Transcripción del documento original del profesor Rapoport realizado para Revista M. English transcription from original document done by professor Rapoport to revista $M$.
The lesson can be about both processes and product characteristics (Rapoport 1989, 1990 c,d). Here I will emphasize product characteristics, supportiveness, open endedness, what I call stable equilibrium (Rapoport 1990 a), pedestrian movement, ambient, complexity, cultural and social responsiveness and so on. (Rapoport $1992 \mathrm{a}$ ). There are also lessons regarding the role of rule systems, which can be implemented using modern materials,

Many of the topics discussed are developed in much more detail in a recent paper "design for people: some implications" (Rapoport 2010)

2 Note, as will be seen later, that this constancy especially of environments is partly a matter of scale - it applies at small rather than larger scale.

3 It may also prove useful to approach them in terms of Patrick Gedde's idea of "conservative surgery "which, unfortunately, has been forgotten and ignored. 
preserving the culture core and providing the appropriate modern image (discussed later) (Rapoport 1983,e, 1994). One can also identify positive and negative environmental quality profiles of different groups, of different actors and understand (and possibly resolve) conflicts (Rapoport 1995, (1990), 2005), it needs to be reiterated that such learning, and the use of built environments on model systems (Rapoport 2006, a) can only occur at some level of abstraction and is never direct (of Rapoport 1987, 1995, a, 1986). (See Figure I)
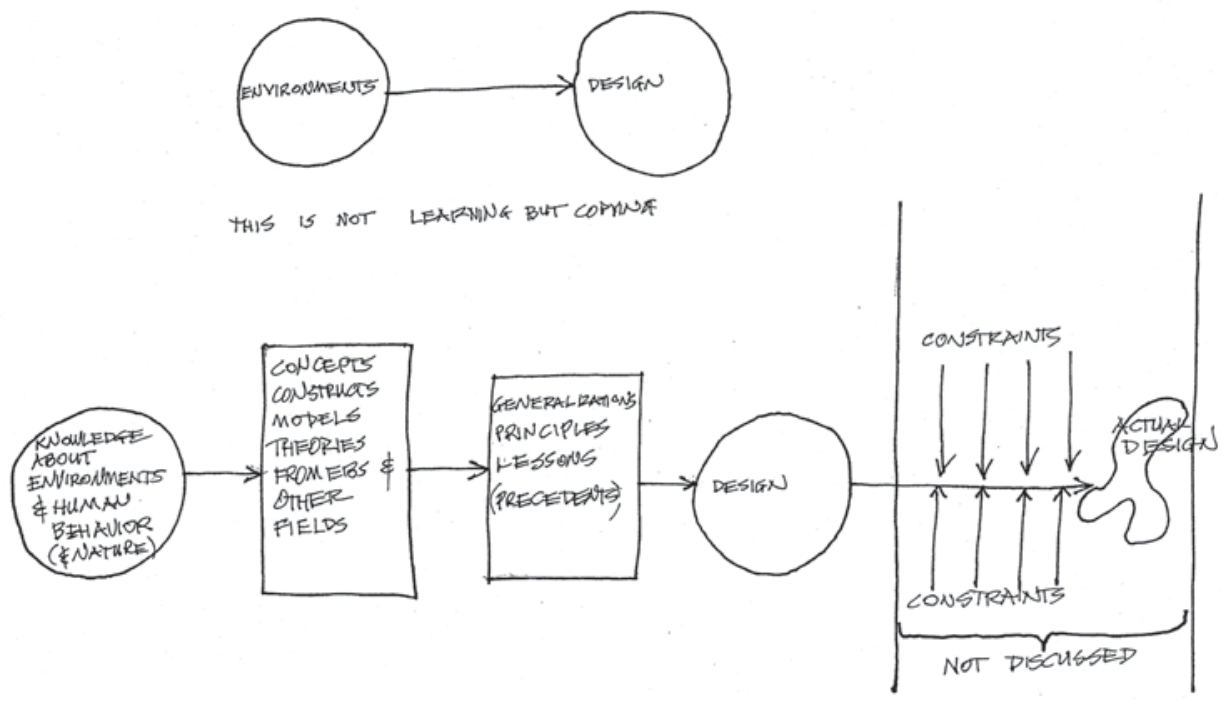

\section{CONSTANCY}

Change, novelty, differences, etc. tend to be emphasized. Looking at constancy might provide a useful corrective.

My emphasis is on research (mainly in EBS seen broadly and as a scientific discipline). Research is fundamental science, and ongoing research is crucial. It provides increasing knowledge which can then lead to evidence-based design (Rapoport 2010).Such research must include the study of the past.

\section{Constancy regarding people}

There is a large and increasing body of work in a number of fields, which emphasizes constancy regarding people, based on their evolution. This work is sociobiology behavioral ecology, evolutionary psychology, primatology and even animal studies suggested that there are human universals, the human nature (ej. Brown 199I) 4 . These work has even begin to influence anthropology (although very slowly) - the example there is a new journal of anthropology and human nature.

This work suggests that culture is not completely variable but has major constancies. Furthermore, it implies that apparently variable aspects may be differing expressions of

$\overline{4 \quad \text { For more details and many more references see (Rapoport 20 }} 10$ )
Figure I. Learning from the full range of environments knowledge of humans. Original Sketch from Amos Rapoport 
universal or constants shared by all cultures. (Brown 1991; goldsmith 1991; pinker 2002; Rhoads 2004, Mclntyre 2006; Cartwright 200I; Alcock 200I; Betzig 1997; Konner 20I0; Barkow et al 1992 among many others).

This view has been reinforced by work of cronk (1999) who uses the concept of an “ ethnographic hyperspace" in this one can visualize a vast number of possible (imaginable) cultures, based on combinations of a restricted number of accepted components of cultures (of From the Human Relations Area Fields). He estimates this number to be 12 followed by 52 zeros, in fact, of course, the number of human cultures is very much smaller. Hauser (2009) makes the same point about both culture and one of its important expressions-language.

It has recently been discovered (Apicella et al 20I2) that social networks among huntergatherers (the hadza, in Tanzania), a proxy for early humans, are the same as current modernized social network. They show remarkable constancy; especially given changes in the scale of society, technology communications, new social institutions etc. Moreover, the explanatory variables are also the same. "Aesthetics" in general (Rapoport 1990b) and I am even more skeptical about its value in environment design which I see as a sciencebased profession not an $\operatorname{art}^{5}$. It is however significant that there is an increasing emphasis on the universality even of aesthetics on the fact that aesthetics preference seem to be valid cross-culturally as an aspect of human nature. These are expressed in culture specific ways (Dutton 2010; Miller 2000; Solso 2003; Zeki I999; among others). A similar view has been expressed about religion (Wade 2010; Lewis-Williams 2010) this no doubt applies to many other aspects of culture.

These developments provide an important corrective to much work on culture and environment (and in the social sciences generally) which, in emphasizing culture and neglecting human nature concentrate only on variability; this corrective has practical implications. It may reduce the variability that are need to consider and deal whit, the numerous small lifestyle of romps (which represents cultures) when dealing with design (Rapoport 2002,2005,2008 c) it may suggest underlying regularities and also help transferability of research alone in one culture to others (Rapoport 2002 b, 2008 a; see Rapoport 2005).

\section{Constancy regarding enviroments}

Since i will be discussing one example (neighborhoods) in some detail later, this section will be brief. There are very many apparently different dwellings around the world; I counted I.278 distinct examples in the encyclopedia of vernacular architecture and that is not a complete coverage (Rapoport 2006 a, p. 179- I80); however, if one considers form rather than shape (Rapoport 1977), then the organization of space and domains, and behavior settings of which all environments are composed (Rapoport 1990 e, 1994 b, 2005). Also I have been looking at, and photographing markets (especially outdoors markets) for decades and find that their organizations, spaces etc, show much constancy (see http: www. sadp.ku.edu/rapoport) this clearly applies to pedestrians space (I 990 c, park III). This may even apply to future cities, which may not be that different - the number of possible cultural landscapes (cities being those most transformed by humans) which are the most useful unit of analysis is limited just as cultures are ${ }^{6}$.

$5 \quad$ Rather than speak of aesthetics it is more useful to think of it as the perceptual, affective and associational aspects of environmental quality, see (Rapoport 2010 and references therein)

6 In the mid 1960's, when I was at the Bartlett, a student analyzed cities as portrayed in science - fiction. He found that there were, for example, many extraordinary technologies described, but all cities were either Le Corbusier's Ville Radiance or Frank Lloyd Wright's Broadacre City. (See Rapoport 1990) 
It is the case that constancy is most evident at small scales, in the case of human settlements at the scale of neighborhoods, rather than cities, metropolises, urban regions etc, (Rapoport 1977, 1995f(1973), 1997, 2002, 2006). At that smaller scale constancy is striking.

At the large scale the contemporary city is different to those of the past. The metropolis has been described as the first new form in 2000 years and clearly megalopolis and urban regions and new forms. But as I have argued for some time (of Rapoport 1977) people do not live in these; they are ethic concepts and constructs. People still live in relatively small areas (sometimes even getting smaller (Rapoport 1997) although how they live in them and use them (may vary) for different groups.

Since neighborhoods are small and often homogeneous along various dimensions (ethnicity, race, tribe, occupation, religion, income, and above all, lifestyle) they are numerous. I have previously cited some figures (Rapoport 1977, ch. 5, esp. pp. 250-255). Recent travels support this. For example in 2009 I visited central Asia and found that the traditional areas of Tashkent, Bukhara and so on are composed of many small neighborhoods. New areas do not seem to have these and I would suggest that contemporary planning and design fail to consider this important aspect of settlement.

These small units can be related to what sociologist call intermediate institutions or mediating_structures these, as will be seen later, play an essential role. In our case these small units are intermediate socio-spatial units between the individual or family and the larger (very much larger) social or urban realm and the most important part of the housesettlement system (Rapoport 1977, 1990 e, 1994 b)

This very strong tendency is also confirmed by ongoing research, as just one example, the traditional part of Kirtipur, a small town in Nepal, has over 30 homogeneous neighborhoods missing in the new areas (Shresthaetal 1997). The city Milwaukee defined and named 186 neighborhoods which, I predicted would be further subdivided (Rapoport 1997). This seems to be happening judging by new names appearing on signs matching those put up by the city. Moreover, ethnic neighborhoods are developing as immigration continues (Decurborn 2008).

Consider an example in lien whit my general argument for the value of using novels, magazines, newspapers, advertisements, etc, (Rapoport $1990 \mathrm{f})^{7}$ in a memoir Estein (2009) points out (p. 3I) that Chicago has 237 neighborhoods(I would predict significantly more, and subdivided as described for Milwaukee). From where people live it was possible to predict ethnicity, income, behavior, space use and institutions. Each neighborhood was effectively a village, defined by ethnicity, folkways, mores, etc. people really left their neighborhood, everything they needed was in them. [This has changes for many, but not all groups (of Rapoport 1977,ch. 5)]. Moreover, as neighborhoods change, they may again become homogeneous, as shown by the different lifestyle groups that can be identified by zip-codes in the US. (Rapoport 1990, 1998, 2000). Moreover the differences between remaining resident and incomers can lead to further subdivisions (of. Suchar and rotenberg 1988 (also dealing whit Chicago). This also establishes new types of homogeneity - - if the principles still apply.

This tendency is also found over time, as revealed by early travel whiting, history and archeology. There is currently much work in archeology showing this, reflecting a growing

7 See also a number of additional examples in (Rapoport 2010) 
interest in such issues. I will just refer to one example (smith 2003). I have also had student papers which reviewed some of the archeological literature and showed the persistence of some neighborhoods; a systematic review of the relevant archeological literature would be most useful ${ }^{8}$.

Thus at the small scale one finds constancy over both time and space suggesting that the presence of these units is highly important. Their basic nature has not changed (although they are less self-contained). They are always present, their size in general similar due to the subjective cognitive definition as socio-spatial schemata (Rapoport 1977, 1997, 2002 c), they tend to be homogeneous even as the criteria for homogeneity vary cross - culturally. Although they do changes, these changes are both much slower and different to those at larger scales (city, metropolis, urban region, etc). These persistent constancies, of both people and environments make possible and help, learning from the past. (As always at the appropriate level of abstraction).

It is also the case that at larger scales the important determinants tend to be economics, technology (of. Traffic), politics, etc. it is at the small scale that human aspects can become dominant. Also in dealing whit the environmental quality of human settlement there will be, in addition to the user, many other actors involved: politicians, business people, economist, engineers, managers, planers and environmental designers (architects, urban designers, landscape architects). It should be pointed out that some aspects of environmental quality cannot be changed by any of the actors, ej. Climate, topography, views, population and social composition etc.

Other components can be changed by politicians (national, regional, local), managers, business people and economists, but not by planers or designers, eg jobs and economy, taxes, school quality, social and urban services (street cleaning, maintenance, garbage collection, water quality, etc). Others yet planners and engineers can influence, but not designers (eg. Public transport, major infrastructure, location of various users and activities, systems of open spaces, overall frameworks, etc). Others are up to users-groups and individuals. Finally there are those things the designers can do, and it is at the small scale that "design" begins to play a role and can, in principle, emphasize the wants and needs of users.

It is useful to identify what distinguishes designers from the other actors, and what environmental designers have in common. It is partly scale, there domain is the middle to small scale. Here they overlap whit users and need to take cultural (group) specificity into account, and maximize open endedness. But there is one aspect witch the other actors (or those who study cities) rarely if ever address the three dimensional (ie. Spatial) and experiential qualities of urban environments, although it is in all sensory modalities (not just visual), it is dynamic, not static and it involves users perception, cognitions and evaluations which need to be discovered. Also, although space organization is clearly necessary it is not sufficient. For example settings need to be considered in connection with activities and behaviors. Also, ambience, the essence of environmental experience depends on semi-fixed and non-fixed elements within the framework of the fixed feature elements (Rapoport 1992, 1993, 2000)

8 Note that archeology is one of the "new" disciplines that EBS needs to consider. It is significant that a number of archeologists have referred to my work, and have become involved whit archaeology (Rapoport 1994c, 1999, 2006) 


\section{THE NATURE OF "DESIGN"}

I put design in scare-quotes because I take a very different view of design which I will only discuss very briefly (of Rapoport 1995 b, 2005, 2010), on this view design need to be seen as research-evidence based, not as on art but a science-based profession. Design need to be seen as hypotheses, based on the best available research, and then tested either by simulation before implementation or by post-occupancy evaluation after implementation and the result feedback into the knowledge base.

In this connection, in addition to formal research method (basic, translational, applied and evaluation) there is the "informal" method, which I have long emphasized the need for it to be used: using the domain as described earlier; using and synthesizing existing knowledge including revisiting earlier work and reinterpreting it in the light of new knowledge; using what I call indirect methods, analyzing non-professional sources briefly mentioned above (of Rapoport I990 f). It is also useful to study environments as one experiences them. This includes local and remote environments as one walks or drives through them or flies over them. This depends on observation, analysis and questions based on prior knowledge, including activities and behavior in these environments semi-fixed elements and changes in environments, their nature, rates of change, including modernization in developing countries, and the effects of migration in both developing and developed countries (rural/urban and international), for example on the formation of ethnic and other enclaves.

Since immigration is stressful, and has been shown to have effects on both mental and physical health, modulating routes of change becomes important (Rapoport I983), in this, small urban units allowing clustering, homogeneity, specific institutions, specific semi-fixed elements and behavior can play a major role. They become supportive for these many groups, allow defensive structuring and also increase the variety of environments. This, in turn increases choice and habitat selection (it also increases the perceptual complexity and richness of cities.

There is one issue specific to developing countries which becomes important since most future urban growth will occur in them. In 1983 (Rapoport 1983) I first pointed out the conflict between the desire for modernity and the need for traditional, familiar, culturespecific, human-need oriented, open-ended settings. Much evidence since has emphasized this issue, which also has an impact on "sustainability" (Rapoport I994 a) and preservation (Rapoport 2002 d). How to reconcile the two becomes an important issue which I will discuss below, in terms of matrix/infil (Rapoport 1992 a, 2000 b).

\section{The role of new (and"unlikely") disciplines- an example}

I have referred briefly to the fact that if approached at same level of abstraction, many new and often apparently "unlikely" fields become relevant. I have for some time added many new mainly scientific fields to the few with which EBS began. (Rapoport 2005, 2008, 2010) ${ }^{10}$.

Here I discuss one example from an extremely "unlikely" field that proved amazingly relevant to my earlier discussion of constancy of people. This was an article about nation-building (mainly regarding Afghanistan, but also referring to Iraq and Pakistan (Etzioni 2009). The concept I discuss is his use of "bottom up" "I. I summarize six general points abstracted from

$9 \quad$ For an extensive discussion see (Rapoport 2010)

10 For a very extensive discussion of many new fields it can be relevant and helpful to refer again to (Rapoport 2010)

II Note that this concept can also be used in other ways which are not as useful for my purposes. It has also been applied to planning; one example is user participation (which is a different albeit important topic in planning and environmental 
the specific context of nation building, I then show how they relate to my discussion above about small urban units and how, together they lead to suggestions about the planning and design of human settlements.

I. Not only traditional societies but even modern so-call mass societies are composed of communities or groups [which Etzioni calls "tribes"]. Individuals are members of many groups - - ethnic, religions, tribal, linguistic, etc / and, I would add, based on sex, age, education and lifestyle). These do not have to be residential [although they can be and often are]. Members of these groups share rules about do's and dont's, habits, beliefs, Rituals, social networks, conflict resolution and so on and on. (These groups correspond to the many small lifestyle groups which I had previously emphasized (Rapoport 1998, 2000 a, 2002 a, 2005, 2008 c).

2. Group membership influences individual choices regarding food, clothing, behavior and so on. There are then regularities within groups and they are not "random". They can be represented by lifestyle and they include environmental choices (and design as a choice process (Rapoport 2005).

3. Ties within groups are stronger than with other groups. Within groups various people - - religion leaders, healers, traders, elite, etc, are highly influential. These internal, or informal, forces and group identity are much stronger than bigger or more formal forces (in this case the national government). I would add that the identity of such groups is often by contrast to others - - "we are not like them". This also leads to defensive structuring with implications for environments (including cities) as the appropriate organizations of space, time, meaning and communication, fixed and non-fixed elements, systems of settings, cultural landscapes and their ambience, clear non-verbal communication etc.

4. It follows that one needs to work with what exists rather than start from scratch, de novo working against it (or fight it), one should direct what exists. (it is significant that Etzioni actually uses the term "design" in connection with, in the case, national institutions). I believe this approach very much applies to the constancies of the small - scale elements in cities, which I have been emphasizing.

5. Elaborating on (4), one should not only use what exists, but also what wants to be. It is easier and more productive to go with the flow, to direct and guide it rather than seek something completely new which is unknown possibly impractical, unacceptable possibly wrong and often rejected ${ }^{12}$.

6. Rather than design an approach or outcome, one should first ask what is the reality on

design). Note also that the "indirect" research method discussed earlier can also be seen as bottom up, in the sense that they provide people's unconscious reactions to, and evaluation of environments.

12 There are often good reasons for what exists, even in apparently simple cases. These need to be understood rather than ignored and replaced. In the 1950's (I do not have the reference) UN agricultural advisors advocated the removal of piles of stone in fields. That made things worse - the stones were trapped in an arid environment. I have discussed comparable examples of such negative unintended consequences in built environments (Rapoport 2005, p. 2-9). There are also many documented cases of the rejection of new housing, with people returning to spontaneous settlements. If the housing is tolerated the results maybe negative. Existing environments suit the ground which developed them and often work well. There are also many resources tied up in them. Saving as much of these environments as possible can have implications regarding "sustainability" (of Geddes "Conservative surgery", the Kampung improvent program in Indonesia, etc) This can also apply to modern areas. When I spent some time in Yogyakarta (Indonesia) I lived in an area of modern "suburban" houses near Gadjah Mada University - that area was entered of a main road (from which it was not visible) and very dearly defined on all sides. Social networks were tight, and the neighborhood organization strong. More traditional kampungs are marked by gates of main arteries, clusters of becaks (bicycle rickshaws) waiting at the gates to negotiate the narrow streets. The result is urbanization in place, and the success of the kampung improvement program - it worked with the existing pattern. 
the ground, what are the existing elements or institutions, how and in which direction they are changing and the extent [and speed] of changes of various elements. And what these might bring (Rapoport 1983; of Rapoport and Hardie 199I).

\section{SOME DESIGN IMPLICATIONS - AN EXAMPLE}

In Human aspects of urban form (1977), in arguing for the importance of small homogeneous urban units, partly because of their persistence cross-culturally and over time, I drew the fallowing diagram (fig. 5.4, p. 263). (Figure 2)

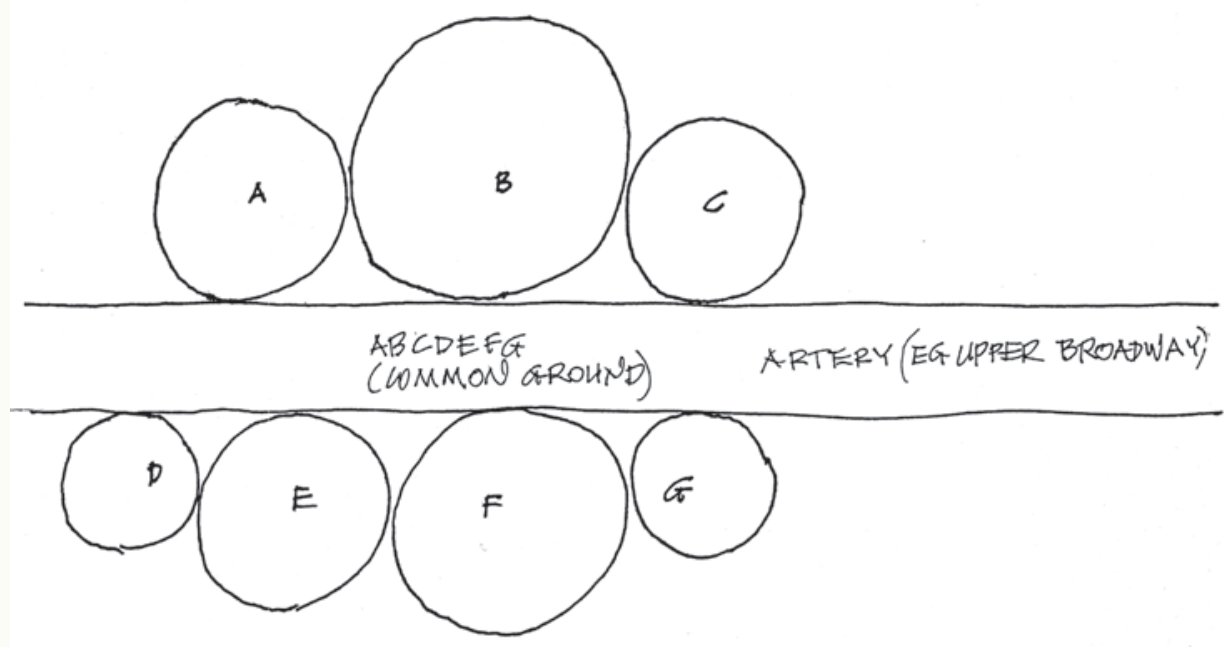

This diagram tried to illustrate the area of upper Broadway in Manhattan. It was highly abstract - New York is not really like that. People, however do try to cluster and to define subjective boundaries of neighborhoods as clearly as possible, and although design can help, the definition is never as clear or distinct as in that diagram. It does, however represent reality in many places. During extensive travels, it has become clear that especially in Asia, but also elsewhere, behind major avenues, with modern office buildings and hotels one finds fully traditional small, tightly defined areas. This is the case in china, India, Thailand, Indonesia, Central Asia, and no doubt elsewhere (Rapoport 1997, 2002 c, 2006 b).

Generalizing: these observations can be generalized and diagrammed as follows (See Figure 3)
Figure 2. Abstract diagram of neighborhood/ artery relation. Original Sketch from Amos Rapoport. 
Figure 3. Actual neighborhood/artery relation as found in India, China, Thailand, Indonesia and more. Original Sketch from Amos Rapoport

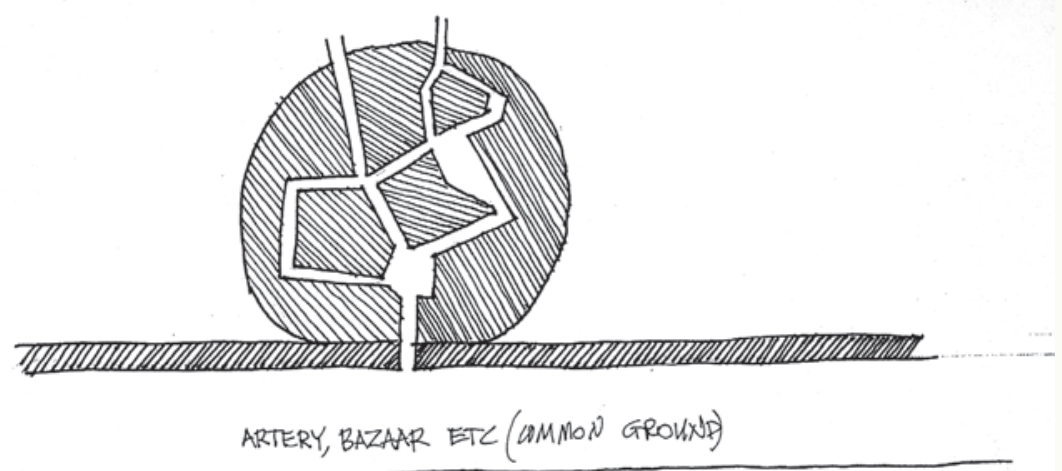

This pattern has many advantages and has much to teach us if we understand it and derive lesson from it (see Image I) ${ }^{13}$. I briefly review some of these advantages.

I. The pattern provides a "front" along the main arteries, the global, expensive modern environment, the appropriate image and the important costly signaling (of Rapaport 2008 b) $)^{14}$. These can respond to technology, economics, politics, traffic, etc.

2. This pattern also provides a "back" region - local tradition spaces, setting for traditional activities, vendors, laundry, playing cards or games, local or games, local meaning, non-verbal cues and institutions. They are culture - specific and if open-ended, allow for personalization by both individuals and groups. Since they can be homogenous such personalization "add up", creating a specific ambience. (Rapoport 1990 b, fig 2 I, p. I38, 1992) These characteristics are important in terms of taking possession, identity, clear cues and easy non-verbal communication.

3. The main arteries constitute "machine space", the back areas provide space appropriate for both dynamic and static pedestrian behavior and slow traffic. Each of this can then provide the appropriate perceptual qualities (Rapoport 1977, ch. 4, esp., 240-247; 1986, I990 c part III, 199I).

4. The ability of these small, local areas to allow for clustering has a number of positive consequences. It locates people in social space, is supportive for different groups, it allows homogeneity to maximize interaction, local rules and non-verbal communication, it is responsive to users in many ways, for example it may allow the operation of different rule systems, and hence different and appropriate site layouts which may be most important culturally (Rapoport 1983,1995 d (1980)) $)^{15}$. These small units are the socio-spatial equivalent of the mediating structures/ equivalent of the mediating structures/ mentioned earlier.

13 It is important to recall that at larger scales (City, Metropolis, Urban, Region) people still live in smaller units of which these settlements consist.

14 The new capital of Turkmenistan (Ashkabad) in an extreme example, being all front, image and "costly signalling". (Rapoport 2008, 20I0) It is highly significant that just I hour away is one of the largest, most traditional markets in central Asia (Tolkuchka).

15 Note that not only is the tendency to cluster ignored, the resulting homogeneity may be prohibited, or at least strongly discouraged. Yet people keep on trying (ie. want it). Thus, in Wisconsin, the proposal for a Hmong town near Green Bay was not allowed; however as we have seen, Hmong have succeeded in some Clustering in Milwaukee (Dearborn 2008) I have also discussed a number of other recent examples (Rapoport 1998, 2000, 2008). One reason given is that clustering will reduce interaction with other groups. This is unlikely since neighborhoods are no longer self - contained. Moreover, going back to the 1960's, having a secure base may in fact increase interaction. There is also what I call "neutral places" in the city as a whole where interaction occurs. The nature of "neutral" may vary in different locales and for different groups - - their provision therefore requires research. 
5. Such small local areas allow open - endedness participation, self - build using different rule systems and thus the creation of appropriate setting and ongoing changes to them. This allows modernization in place so that what people can do is not only allowed but encouraged (lesson of spontaneous settlements). Changes can occur within neighborhoods independently of other and without disturbing the larger urban systems; the framework and the appropriate image can remain unchanged or can change responding to the different forces already discussed.

6. The presence of clearly demarcated small units can also help responses at this larger scale. For example, it becomes easier to "thread" e.g. Highways between neighborhoods, not thorough them, maintaining their integrity and also important links among them. (Figure 4)
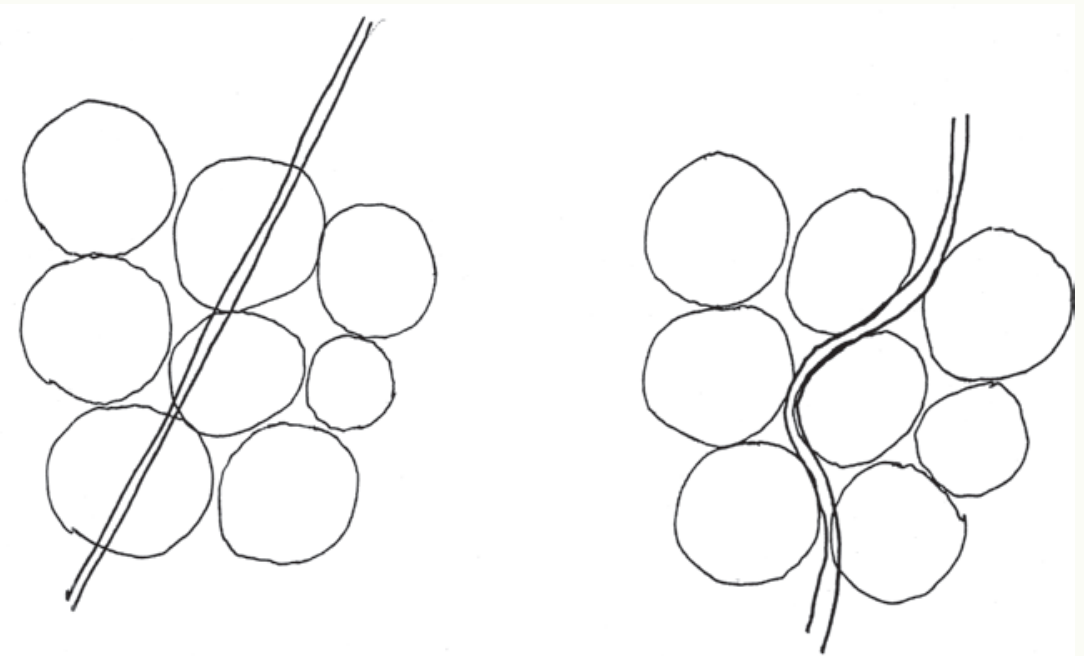

7. Within the small areas one often finds modernity gradients, the most modern at the entry and the least modern at the "back". This allows for gradual acculturation and ongoing modernization in place. Modern materials, services, etc, can be introduced gradually to improve material condition without losing other advantages, of course, this happens naturally in spontaneous settlements (eg. In Latin America) and can be directed as in the Kampung improvement program in Indonesia. The process can go on as cultural change continues, and environmental quality expectation rise (eg. Sastrosasmitaand Nurul Amin 1996).

Within these areas one also finds penetration gradient with some measure of control over the group territory. This has implications for self-policing and safety.

I have already mentioned that many of the above characteristics help reduce stress caused by migration, culture damage and organization. These processes have also been shown to increase the incidence of mental illness, eg. Schizophrenia. Homogeneous and responsive areas reduce this incident. They also mitigate the effects of urban environments generally in which Schizophrenia is more prevalent than in rural environments. (Van Os, et. al. 2010)16. ${ }^{17}$

10. The small units whit the characteristics described in I -- 9 take on specific ambience, identity etc, which can change in response to changes in population common in all cities. New groups can transform the units, further increasing variety. O'donell (1995) looked at
Figure 4. Alternative highway routes in an urban area. Original Sketch from Amos Rapoport

16 It should be noted that in all cases genetic predispositions play a role. 
Figure 5. Graphic summary of implications of argument. Original Sketch from Amos Rapoport

a small area of lower Manhattan, beginning in the $17^{\text {th }} \mathrm{C}$, while the mayor streets remained unchanged, there were major changes in land subdivision, activities and settings specific to the successive different groups that lived there. More specifically he identified a recent, and ongoing, change from "Little Italy" to Chinatown, whit changes in uses and ambience (via semi-fixed elements, colors, smells, sounds and activities). By analyzing the changes within that small area, o'donnell suggested how open-endedness could be increased.

Also these small units discussed in I-- I I are important not only for the inhabitants enabling them to identify with areas, use them for defensive structuring etc. They also create a great variety of habitats, with different ambiences, different organizations of space, time, meaning and communication, colors, sounds, smells, semi-fixed elements etc. this makes the city as a whole much richer and more complex, perceptually. It also maximizes choice among habitants and choice is an important aspect of the effect of environment on behavior (Rapoport 2005, pI I). By analogy with ecology, one can argue that the perseveration of a variety of habitats (habitat heterogeneity) which preserves biological diversity may also preserve cultural diversity, the loss of which may be like the loss of bio- and eco-diversity. It also preserves a variety of environments for further study and further lessons.

The points discussed above, in I-- 10 can be partly summarized in a single diagram. (See Figure 5)

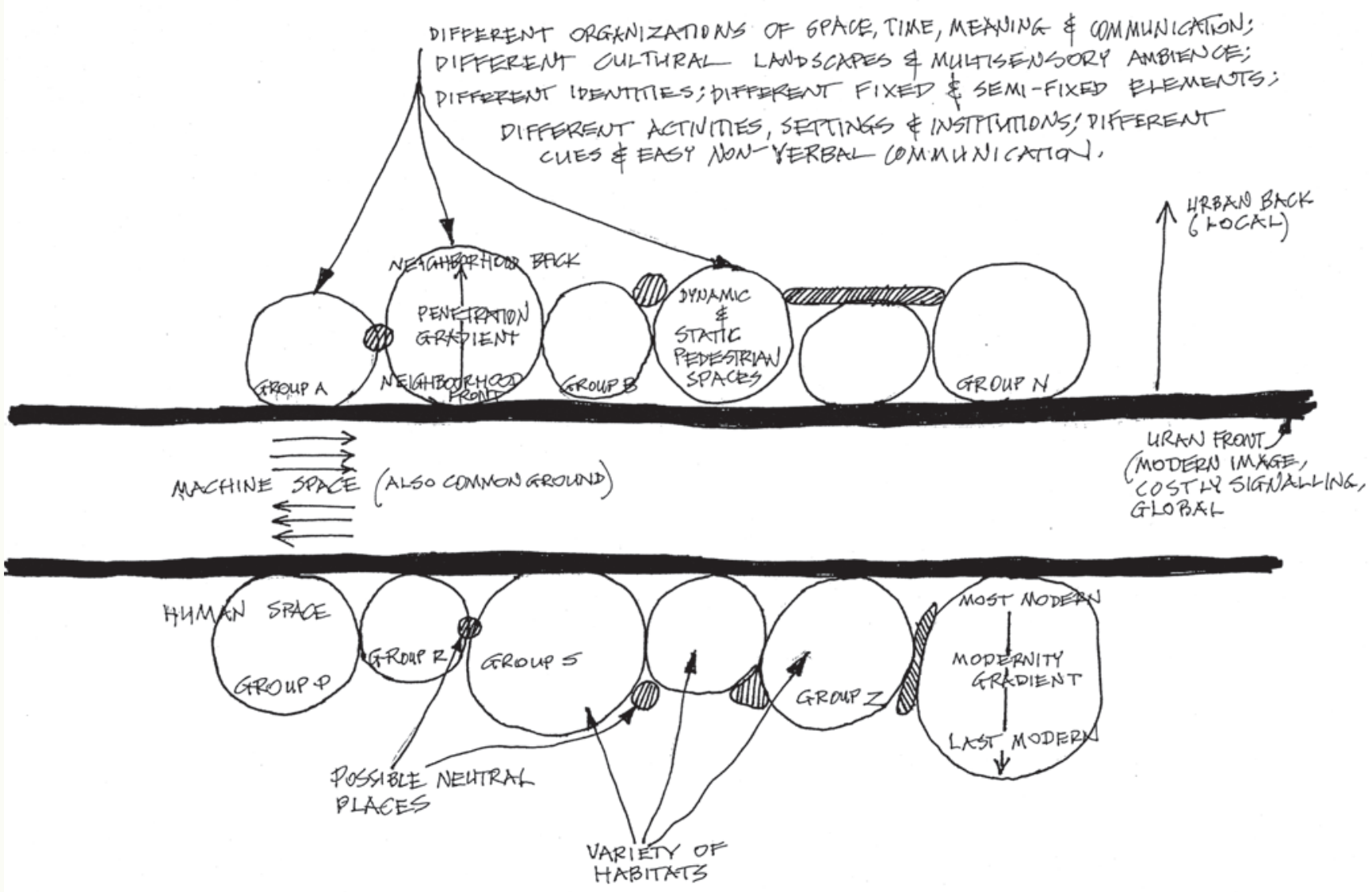




\section{CONCLUSION}

One can understand many cities, and cultural landscapes in general in terms of a (often high - style) matrix (Rapoport 1992 a) and can be related to Bacon's (1967) view of cities and the urban version of Habraken's frame (of Rapoport 1995 c, (1990/9I)). What I have described as machine space, environments responding to economic, technological, political and other forces form the matrix. The small units I have emphasize and described constitute the infill. There is an element of constancy even at the larger scale. This approach, therefore, capitalizes on what is there, what is happening and "wants" to happen. This makes it easier to implement, control, direct and regulate than imposing de novo solutions that may not even work.

It is here that Etzioni's (2009) nation building approach and my approach to human settlements overlap. The parallels are amazing. Conceptually, the conclusions from these two very different domains are remarkably similar and reinforce each other. This paper is almost like a physical version, at the different scale and in a different domain of Etzioni's argument, showing the value of using a variety of fields, if approached properly.

Considered broadly, one seems to find two conflicting tendencies. On the one hand there is global change seemingly leading to the blurring of distinctions and reductions of differences, a universal urban landscape. On the other hand one finds a growing number of lifestyle groups, an increasing search for cultural, ethnic, linguistic local and regional identities. These two trends, and the conflict between them, can possibly be resolved by the framework approach. The frameworks can be similar everywhere projecting the desired images. It can respond to all the differences (including the apparent quest for the "suburban" image) and with the advantages described. It can allow different rule systems, different identities, provide human spaces and appropriate perceptual qualities, respond to the growing impact of migration, both rural and urban and international.

The argument of this paper is of particular value in developing countries, where most urban growth will take place. But it is also relevant in developed countries with the growing number of lifestyle groups, increasing choice and weaker constrains, international migration and the persisting, and even increasing, importance of neighborhoods.

It is, however the study of constancies in both, people and environments which makes possible learning from the past (and present) and applying these lesson to the future.

\section{ADDENDUM}

Since completing the last draft of these papers, some new material has come to hand. It reinforces the important points made in the paper. First, the importance of keeping up whit ongoing research in various fields and the utility of popular media. Second, that once conceptual and theoretical frameworks are developed, not only to new findings fit in to them but it is possible easily to "read" environmental and show how they both fit and reinforce the argument.

Regarding the first point, several references can usefully be added: these follow. Regarding the second I describe a visit to a village in Yunnan, China.

I. A. 4 papers showing the use of constancy at different scales, in different places and concerning different topics. (Open - ended design, sustainability, comfort and cultural appropriateness). All are in one issue of Open house international, Vol. 36, No 4 (20I I)

M 33 REVISTA M VOL. 9 No.1. ENERO-JUNIO 2012 • FACULTAD DE ARQUITECTURA • UNIVERSIDAD SANTO TOMÁS COLOMBIA 
Figure 6. Xingmen Village. Original Sketch from Amos Rapoport (i) BAIGO, T. et. al. "Future directions and sustainable buildings in Japan". p. 5-19. This is based on an analysis of traditional Japanese houses.

(ii) BEISI, J., and J. LINGYING. "Flexibility of traditional Buildings and craftsmanship in China”. p. 20-3I.

(iii) NA, L.T.H and J-H., PARK. "Applying eco-features of traditional Vietnamese houses to contemporary high - Rise housing”. p. 32-45.

(iv) AL-AMARECH, A. "The Bedouin tent in comparison with UAE housing provision”. This uses and analysis of the traditional Bedouin winter tent to evaluate government housing and suggest improvements related to cultured patterns and behaviors. p. 82-97.

B. An as yet unpublished book in archaeology from the University of Arizona Press: Arnauld, M.C et.al. (Eds) The Neighborhood as a social and spatial unit in Mesoamerican Cities. This echoes and supports my work on neighborhoods and their importance generally (of. Rapoport 1997, 2002 in References to this papers) for the case of Mesoamerica. This is based on the availability of new research in that region and also relates the material to similar findings all over the world. (Of Smith (2003) in references to this papers. Of also Calpulli in Tenochtitlan in Aguilar - Moreno, M (2006) Life in the Aztec World, Oxford University press)

C. Newman, M (2012) "Terraced living in a San Diego Quarry "New York Times (Feb 29). This describes plans for a relatively new and rapidly growing US. City. The Objective is to turn San Diego into a "city of Villages" (of E.s. Rasmussens early book on London as

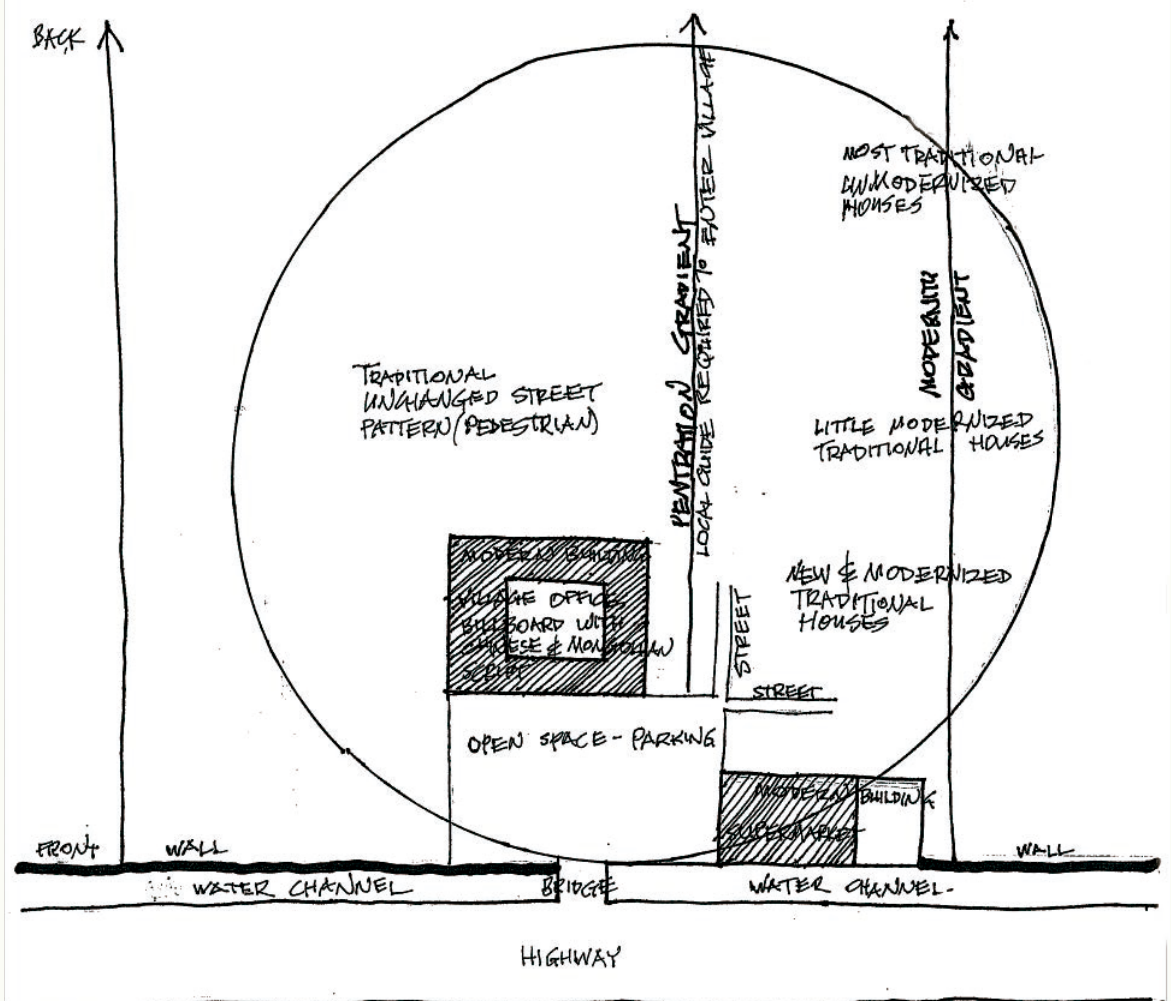

REVISTA M VOL. 9 No.1. ENERO-JUNIO 2012 • FACULTAD DE ARQUITECTURA • UNIVERSIDAD SANTO TOMÁS COLOMBIA M 34 
a city of villages). From the description these villages correspond to the units illustrates in Image 5 in this paper. Also emphasized in the possibility as a pedestrian orientation in these units (Of Figures 3 and 5 in paper and Rapoport 1990 in the references there in.)

2. On April 8, 20I2, during a trip to SW China visiting various minority groups I visited Xingwen village in Yunnan province. This is a village of Mongolian who has been there since the time of Genghins Khan $\left(13^{\text {th }} \mathrm{c}\right.$.) They speak both Mongolian and mandarin and use both scripts on signs. This shows the persistence of ethnic groups both in China and elsewhere (of Rapoport (200a, 2002a) and Dearborn (2008), in references in my paper. Of also Pagel, M (20I2) Wired for Culture, New York, W.W. Norton).

Physically, the village is an almost perfect example of the Characteristics of the units show in Figure 5 in my paper. (An analysis in Figure 6)

\section{BIBLIOGRAFÍA / BIBLIOGRAPHY}

ALCOCK, J. The triumph of sociobiology. Oxford: Oxford University Press, $200 \mathrm{I}$.

APICELLA, C.L, et.al. "Social networks and cooperation in hunter-gatherers". In Nature, Vol. 48I, Issue 7382, January. 2012

ATRAM, S. In Gods we trust (The evolutionary landscape of religión). Oxford: Oxford University Press, 2002.

BACON, E.N. Design of cities. New York: Viking Press, 1967.

BARKOW, J.W., et.al. The adapted mind (evolutionary psychology and the generation of culture. New York: Oxford University Press, 1992.

BETZIG, L. (Ed.). Human nature:a critical reader. New York: Oxford University Press, 1997.

BROWN, D.E. Human Universals. Philadelphia: Temple University Press, I99I.

CARTWRIGHT, J. Evolution and human behavior. London: Palgrave, $200 \mathrm{I}$.

CRONK, L. That complex whole (culture and the evolution of human behavior). Boulder CO:Westview Press, 1999.

DEARBORN, L.M., "Reconstituting among cultura and traditions in Milwaukee,

WISCONSIN". In: Traditional dwellings and settlements review. Vol. 19, No. 2. Spring, 2008.

DUTTON, D. "The art instinct". Beauty, pleasure and human evolution. New York: Bloomsbury Press, 2010.

EPSTEIN, J. “Toddling town”. In: Commentary. Vol. I28, No. 4. November. 2009.

M 35 REVISTA M VOL. 9 No.1. ENERO-JUNIO 2012 - FACULTAD DE ARQUITECTURA • UNIVERSIDAD SANTO TOMÁs COLOMBIA 
ETZIONI, E. “Bottom-up nation building”. In: Policy Review. No I58. December. 2009.

GOLDSMITH, T.H., The biological roots of human Behavior. New York: Oxford University Press, 1991.

HAUSER, M.D. "The possibility of imposible cultures”. In Nature, Vol. 460, Issue 7252, July. 2009.

KONNER, M. The evolution of childhood. Cambridge, MA: Belknap Press of Harvard University, 2010.

McINTYRE, L. Dark ages (the case for a science of human behavior). Cambridge, MA.: Bradford Books/MIT Press, 2006.

MILLER, G. The mating mind. New York: Doubleday, 2000.

O'DONELL, S. Towards urban framework (accommodating change in urban cultural landscapes). Milwaukee, WI: Dept. of Architecture. M. Arch Thesis (unpublished)

PINKER, S. The blank slate (the modern denial of human nature). New York: Viking, 2002.

RAPOPORT, A. Human aspects of urban form, Oxford: Pergamon Press, 1977.

RAPOPORT, A. “Development, culture change and supportive design”. In: Habitat International. Vol. 7 No. 5/6. 1983.

RAPOPORT, A. "The use and design of open spaces in urban neighborhoods". In: FRICK, D. (Ed.) The quality of urban life (social, psychological and physical conditions). Berlín: De Gruyter, 1986.

RAPOPORT, A. "Learning about settlements and energy from historical precedents". In: Ekistics.Vol. 54, Nos. 325-327 (July-Dec). 1987.

RAPOPORT, A. "Spontaneous settlements as vernacular design” in C.V. Patton (ed.) Spontaneus Shelter. Philadelphia, Temple University Press. 1988.

RAPOPORT, A. "On the attributes of tradition”. In J-P. Bourdier and N. Aesayyad (eds.) Dwellings, settlements and tradition, Lanham, M D. University Press of America. 1989.

RAPOPORT, A. “Defining vernacular DESIGN”. In: TURAN, M. (Ed.) Vernacular architecture (Paradigms of Environmental Response. Aldershot. (UK). Avebury. 1990a.

RAPOPORT, A. The meaning of the build environment. Tucson: University of Arizona Press, 1990b.

RAPOPORT, A. History and precedent in Environmental Design, New York. Plenum, 1990c.

RAPOPORT, A. "Levels of meaning and types of environments". In Y. YOSHITAKE et.al. (Eds.) Current Issues in Environment-Behavior Research. Tokio, University of Tokio. 1990d. 
RAPOPORT, A.”Systems of activities and systems of settings". In: KENT, S. (Ed.) Domestic architecture and the use of space. Cambridge: Cambridge University Press, 1990e.

RAPOPORT, A. "Indirect approaches to environment-behavior research". In: The National Geographical Journal of India. Vol. 36 (parts I and 2) March/June, I990f.

RAPOPORT, A. "Pedestrian street use: culture and perception". In: VERNEZ

MOUDON, A. (Ed.) Public streets for public use.New York: Columbia University Press, 1991.

RAPOPORT, A. "On cultural landscapes". In: Traditional Dwellings and Settlements Review. Vol. 3, No 2. (Spring). 1992a.

RAPOPORT, A. "On regions and regionalism". In: MARKOVICH, N.C., PREISER, W.F.E, and STURM, F.G. (Eds.) Pueblo style and regional architecture. New York: Van Nostrand Reinhold, 1992b. (paperback edition only).

RAPOPORT, A. Cross-cultural studies and urban form.(The 1992 Lefrak Lectures) College Park, MD, University of Maryland, Urban Studies and Planning Program. 1993a.

RAPOPORT, A. Cross-cultural studies and urban form.(The 1992 Lefrak Lectures), College Park, MD, University of Maryland, Urban Studies and Planning Program. 1993a.

RAPOPORT, A. "On the nature of capitals and their physical expression". In: TAYLOR, J., et.al.(Eds.) Capital cities: International perspectives. Ottawa: Carleton University Press. 1993b.

RAPOPORT, A. Sustainability, Meaning and Traditional Environments. Berkeley, CA. Center for Design Research. University of California. Traditional Dwellings and Settlements Working Paper Series. Vol 75. 1994a.

RAPOPORT, A."Space organization and the built environment”. In: INGOLD, T. (Ed.) Companion enciclopedia of antropology. London: Routtedge, 1994b.

RAPOPORT, A."On my relationship to archaeology”. In: Section News, Archaeology Division, Anthropology Newsletter, December. 1994c

RAPOPORT, A. "Settlements and energy: historical precedents" In: RAPOPORT, A. ThirtyThree Papers in Environment-Behavior Research. Newcastle (UK). Urban International Press. 1995a.

RAPOPORT, A. "On the nature of design”. In: Practices. Issue 3/4, Spring. 1995b.

RAPOPORT, A. "Flexibility, open-endedness and design". In: RAPOPORT, A. Thirty-Three Papers in Environment-Behavior Research. Newcastle (UK). Urban International Press. 1995c.

RAPOPORT, A. "Culture, site layout and housing". In: Thirty-Three Papers in EnvironmentBehavior Research. Newcastle (UK). Urban International Press. 1995d. [1980].

M 37 REVISTA M VOL. 9 No.1. ENERO-JUNIO 2012 • FACULTAD DE ARQUITECTURA • UNIVERSIDAD SANTO TOMÁS COLOMBIA 
RAPOPORT, A. ."Environmental quality and environmental quality profiles". In: RAPOPORT, A. Thirty-Three Papers in Environment-Behavior Research. Newcastle (UK). Urban International Press. 1995e.

RAPOPORT, A."The city of tomorrow, the problems of today and the lessons of the past". In: RAPOPORT, A. Thirty three papers in Environment-Behaviour Research". Newcastle (UK): Urban International Press, $1995 \mathrm{f}$ [1973].

RAPOPORT, A. "The nature and role of neighborhoods". In: SHOKOOHY, M. (Ed.) Urban Design Studies. Vol 3. London: School of architecture and landscape, University of Greenwich, 1997.

RAPOPORT, A. "Using 'culture' in housing design". In: Housing and Society. Vol. 25, No. I. (Outsummer 1999). 1998.

RAPOPORT, A. "Archaeological inference and environment-behavior studies". In BRAEMER, F., et. al. (Eds.) Habitat et societ. Antibes: Editions AFDCA, 1999.

RAPOPORT, A. "Theory, culture and housing". In: Housing, Theory and Society. Vol. 17 No. 4. 2000a.

RAPOPORT, A. "On the perception of urban landscapes". In: Urban Design Studies. Vol. 6. London: School of Architecture and Landscape. University of Greenwich, 2000b.

RAPOPORT, A."On the size of cultural groups". In Open house international, Vol. 27, No. 3, September, 2002a.

RAPOPORT, A."Environment-behavior research in an Asian-Pacific context". In Journal of Asian Urban Studies. Vol.3, No, 3. March. 2002b.

RAPOPORT, A. "The role of neighborhoods in the succes of cities". In: Ekistics. Vol. 6, No 4I5-4I7. July-Dec, 2002c.

RAPOPORT, A. "Traditional environments, culture and preservation". In: TURGUT, $\mathrm{H}$. and KELLET, P. (Eds.) Traditional environments in a new millennium. Istanbul: Istanbul Technical University IAPS-CSBE. 2002d.

RAPOPORT, A. Culture, Architecture and Design. Chicago. Locke Scientific. 2005.

RAPOPORT, A. ."Vernacular design as a model system”. In: ASQUIT, L. and VELLINGA M. (Eds.) Vernacular achitecture in the 2 I st century:Theory, education and practice. Abingdon, Oxon (UK): Taylor and Francis, 2006a.

RAPOPORT, A. "Local environments in a global context”. In: Ekistics. Vol. 73, No. 43644I. Jan-Dec. 2006b.

RAPOPORT, A. "Archaeology and environment-behavior studies". In: ASHONORE, W., et. al. (Eds.) Integrating the diversity of twenty-first century anthropology. No. 16. Berkeley, CA.: Archaelogical paper of the American Antrophological Association, 2006c. 
RAPOPORT, A. "Environment-behavior studies: past, present and future". In: Journal of architectural and planning research. Vol. 25, No. 4. Winter. 2008a.

RAPOPORT, A. "Mind, land and society ina an environment-behavior perspective". In: Arquitectonics I5. Barcelona: Ediciones UPC. 2008b.

RAPOPORT, A. "Some further thoughts on culture and environment". In: International Journal of Architectural Research. Vol. 2, Issue I. March. 2008c.

RAPOPORT, A. "Designing for people: some implications" In: TRIALOG, No. 106. March, 2010.

RAPOPORT, A. and HARDIE, G. "Cultural change analysis: core concpets of housing for the Tswana". In: TIPPLE, A. G. and WILLIS, K.G. (Eds.) Housing for the por in the third world (Methods, analysis, case studies and policy). London: Routledge, 1991.

RHOADS, S.E. Taking sex differences seriously.San Francisco: Encounter Books, 2004.

SASTROSASMITA, S. and MURUL AMIN, A.T.M. "Housing needs of informal sector workers: the case of Yogyakarta, Indonesia”. In: Habitat International. Vol. I4 No. 4. 1996.

SHRESTA, U. S., et. al. "Social effects of land use change in Kirtirpur, Nepal". In: SHOKOOHY, M. (Ed.) Urban design studies. Vol. 3. London: School of architecture and landscape. University of Greeenwich, 1997.

SMITH, M.L. (Ed.) The social construction of ancient cities.Washington, D.C.: Smithsonian Books, 2003.

SOLSO, R. L. The psychology of art and the evolution of the conscious brain. Cambridge, MA: MIT Press, 2003.

SUCHAR, C.S. and ROTENBERG. "Judging the adequacy of shelter: a case from Lincoln Park". Paper presented at the meeting for applied antropology. Tampa, Florida. (Mimeo), 1988.

VAN OS, J., et al. "The environment and schizophrenia". In: Nature, Vol. 468, Issue 732 I November, 2010.

WADE, N. The faith instinct, New York: Penguin Books, 2010+ LEWIS-WILLIAMS,

D. CONCEIVING GOD. The cognitive origins and evolution of religión. London: Thames \& Hudson, 2010.

WRIGHT, R. The moral animal (Evolutionary Psychology and Everyday Life).New York: Vintage Books, 1994.

ZEKI, S. Inner visión (an exploration of art and the brain). Oxford: Oxford University Press, 1999.

M 39 REVISTA M VOL. 9 No.1. ENERO-JUNIO 2012 • FACULTAD DE ARQUITECTURA • UNIVERSIDAD SANTO TOMÁs COLOMBIA 